\title{
Birds of the Lobéké Faunal Reserve, Cameroon, and its regional importance for conservation
}

F. DOWSETT-LEMAIRE and R. J. DOWSETT

\begin{abstract}
Summary
The Faunal Reserve of the Lobéké area of south-eastern Cameroon is an important area for forest conservation. One day, it is hoped, it will be part of an international unit, in association with two protected areas in neighbouring countries (Dzanga-Ndoki National Park, Central African Republic and Nouabalé-Ndoki National Park, Congo). With a view to assessing the status of its avifauna in particular, 24 days were spent in three short surveys from 1997 to 1999 . The main forest type is semi-evergreen, with an open canopy; the only natural savannas are small saline swamps. The total of 305 species of bird recorded includes a forest nightjar not yet identified (but more likely to be the rare Itombwe Nightjar Caprimulgus prigoginei than a new species) and the Dja River Warbler Bradypterus grandis. The latter is a species confined to Rhynchospora swamps and had not been re-located in Cameroon since it was first collected in 1914 west of the Dja river; the extent of suitable habitat in Lobéké makes this site the most important to date for its conservation. Other rare or little-known forest species recorded include Olive Ibis Bostrychia olivacea, Sandy Scops Owl Otus icterorhynchus, Zenker's Honeyguide Meligomon zenkeri, Tessmann's Flycatcher Muscicapa tessmanni and Yellow-capped Weaver Ploceus dorsomaculatus. Barred Owlet Glaucidium capense is locally common in open-canopy forest: this population was only recently discovered in central Africa and its taxonomic relationships have yet to be determined. We include a brief comparison with the avifauna of adjacent Dzangha-Ndoki National Park and Nouabalé-Ndoki National Park. A feature of the Lobéké avifauna is the presence of a few species normally associated with forest at higher altitudes (such as White-headed Wood Hoopoe Phoeniculus bollei, Uganda Woodland Warbler Phylloscopus budongoensis, Black-throated Apalis Apalis jacksoni), and perhaps absent from lower-lying Nouabalé-Ndoki.
\end{abstract}

\section{Introduction}

The Lobéké Faunal Reserve (Réserve de Faune de la Lobéké) covers some 2,00o $\mathrm{km}^{2}$ in the extreme south-east of Cameroon. The area originally protected legally was $413 \mathrm{~km}^{2}$, centred on "Lac Lobéké", but it is intended to extend this area, although the precise limits are not yet finalized. It is probable that these will run southwards from the Lobéké river and east to the Sangha river, which forms the frontier with Central African Republic (or RCA) and Congo. The southern and western limits remain to be decided, but they will not reach the inhabited zones along the Ngoko river and the road between Yokadouma and Moloundou, contrary to the map presented by Smith et al. (1996). The Reserve as presently under- 
stood (Figure 1) covers a forest devoid of permanent habitation; however, it was crossed from west to east by an important forestry road, which reached Djembé on the Sangha (the port handling logs transported from Congo), with two axes to the south, to Kika on the Ngoko river and Socambo-Mawa on the Sangha. The Djembé road was closed in late 1997 and is now disused. Timber exploitation has never affected more than a small part of the Lobéké Reserve, because it was limited to areas along forestry tracks, and operations were recently halted. Similarly, safari hunting (including elephant hunting), which was carried out in several small, swampy savannas in the Reserve until 1996, has also been transferred now to regions outside the Reserve. Nevertheless, poaching of large mammals and of certain birds (especially parrots) is widespread and difficult to control.

We first visited Lobéké from 13 to 25 April 1997, to undertake an avifaunal survey for WWF-Cameroon. The Lobéké area's avifauna was very incompletely known and, in order for its importance to be assessed nationally (e.g. for BirdLife International's Important Bird Areas project) and regionally (for the planned association with neighbouring parks in RCA and Congo), a more thorough survey was necessary. Indeed, more practically, the limits of the planned extension to the Lobéké Reserve will be determined by the surveys (of wildlife and vegetation) that are underway. Further brief ornithological surveys were continued in December 1997 and April 1999.

\section{Study area}

According to map sheet Abong Mbang NA-33-N.O. (1:500,00o), the altitude of the Reserve varies from $336 \mathrm{~m}$ (on the Sangha) to about $750 \mathrm{~m}$ (a fairly prominent ridge between the Lobéké and Boulou rivers is above the $700 \mathrm{~m}$ contour). Operational Navigation Chart ONC L-3 indicates also two small hills in the Reserve which reach $824 \mathrm{~m}$.

The main forest type is semi-evergreen Sterculiaceae forest with an open canopy (cf. Letouzey 1968). Dominant species of large tree (40 $\mathrm{m}$ and more) are Triplochiton scleroxylon, Pterygota macrocarpa (two Sterculiaceae), followed by Ceiba pentandra (Bombacaceae) and Terminalia superba (Combretaceae). The understorey, some $4-5 \mathrm{~m}$ high, is very dense and dominated by Marantaceae (notably Haumania danckelmaniana, a creeping species with spines) and Zingiberaceae. Near certain streams and in areas slightly marshy or liable to flooding the herbaceous understorey is replaced by a more closed woody understorey, some 6-8 $\mathrm{m}$ high; Ebenaceae (Diospyros) and Annonaceae (Meiocarpum) are dominant. The second important type of forest is evergreen, dominated by Gilbertiodendron dewerrei (with closed canopy), which exists in small pockets here and there, notably near certain streams such as on the Boulou river.

The only natural savannas are swamps, and the reserve has more than a dozen important marshy areas, of up to $2-3 \mathrm{~km}^{2}$ in extent. The one at Boulou is essentially a dambo, with seasonally flooded grassland (Echinochloa pyramidalis) around a wetter central section with sedges (Eleocharis). Most of the others are rather saline, and important sections are occupied by the tall, leafy sedge Rhynchospora corymbosa. The largest marsh we visited ("Lac Lobéké" or Djangé) is $c$. $1.5 \mathrm{~km}$ long, although it is not the most extensive in the area. This marsh has 


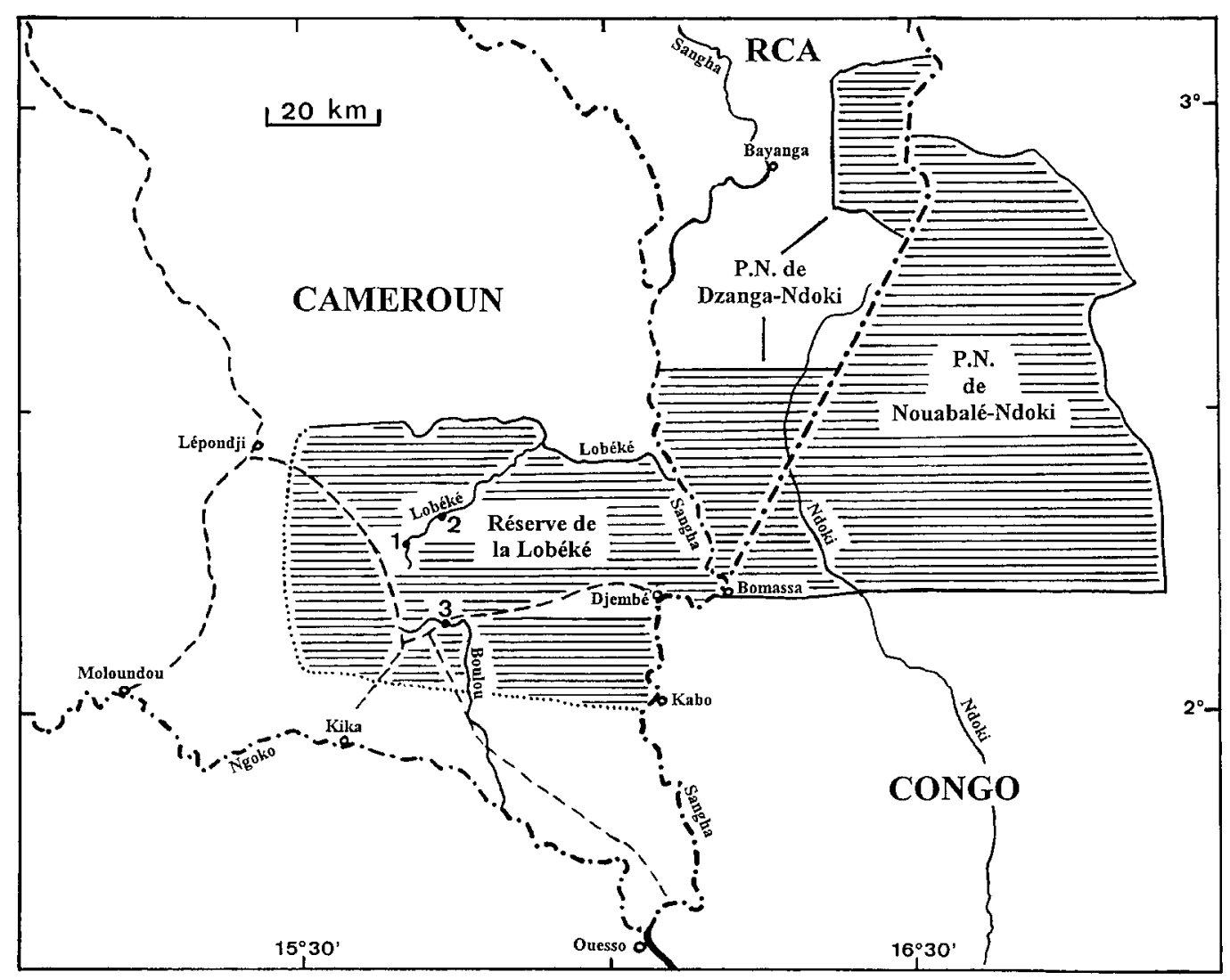

Figure 1. Map of Lobéké Faunal Reserve and adjacent national parks in Congo and Central African Republic (RCA). South-western limits of the reserve are approximate; dirt roads are shown as interrupted lines; 1, Lobéké camp; 2, Djangé ("Lac Lobéké") marsh; 3, Boulou camp. 
been called a "vast swamp ..." by Lamarque et al. (1990), which seems inappropriate. The vegetation around the edges is dominated by thickets of the palm Phoenix reclinata, mixed with species such as Alstonia congensis, Berlinia sp., Lophira alata, Rauvolfia caffra. In the flooded areas there are raphia thickets (Raphia sp., perhaps mombuttorum) and copses of Pandanus. The wide Sangha river and its sandbars constitute an important habitat for a variety of waders and pratincoles, especially in the dry season.

We give below the coordinates of the different localities mentioned in this paper (see also Figure 1). These are in Cameroon, unless otherwise stated, and most were verified with a G.P.S.:

Bityé:

Bomassa (Sangha, Congo side):

Boulou, camp:

Boulou, Marsh:

Djembé (Sangha):

Ibembo (Zaire):

Kupandaka swamp (Nki buffer zone):

Lobéké, camp:

Lobéké, first marsh ("Petite savane"):

Lobéké, "Lac" or second marsh ("Djangé"):

Mala swamp (Nki Faunal Reserve):

Mbéli bai (Congo):

Moloundou (Ngoko river):

Ndoki Camp (Congo):

Ngotto (RCA): $3^{\circ} \mathrm{O} 1^{\prime} \mathrm{N}, 12^{\circ} 22^{\prime} \mathrm{E}$
$2^{\circ} 12^{\prime} \mathrm{N}, 16^{\circ} 11^{\prime} \mathrm{E}$
$2^{\circ} 09^{\prime} \mathrm{N}, 15^{\circ} 44^{\prime} \mathrm{E}$
$2^{\circ} 10^{\prime} \mathrm{N}, 15^{\circ} 43^{\prime} \mathrm{E}$
$2^{\circ} 11^{\prime} \mathrm{N}, 16^{\circ} \mathrm{O} 4^{\prime} \mathrm{E}$
$2^{\circ} 38^{\prime} \mathrm{N}, 23^{\circ} 37^{\prime} \mathrm{E}$
$2^{\circ} 1^{\prime} \mathrm{N}, 14^{\circ} 51^{\prime} \mathrm{E}$
$2^{\circ} 17^{\prime} \mathrm{N}, 15^{\circ} 40^{\prime} \mathrm{E}$
$2^{\circ} 17^{\prime} \mathrm{N}, 15^{\circ} 42^{\prime} \mathrm{E}$
$2^{\circ} 19^{\prime} \mathrm{N}, 15^{\circ} 45^{\prime} \mathrm{E}$
$2^{\circ} 12^{\prime} \mathrm{N}, 14^{\circ} 39^{\prime} \mathrm{E}$
$2^{\circ} 12^{\prime} \mathrm{N}, 16^{\circ} 24^{\prime} \mathrm{E}$
$2^{\circ} \mathrm{O} 2^{\prime} \mathrm{N}, 15^{\circ} 13^{\prime} \mathrm{E}$
$2^{\circ} 12^{\prime} \mathrm{N}, 16^{\circ} 23^{\prime} \mathrm{E}$
$3^{\circ} 50^{\prime} \mathrm{N}, 17^{\circ} 20^{\prime} \mathrm{E}$

March-April marks the start of the rainy season, the driest months in this region being December to February. Rainfall was relatively heavy at Lobéké in April 1997 (much less so on the Congo side of the Sangha), with a substantial storm on average every other day; in 1999 the rains had been delayed and were just starting in April.

\section{Methods}

During our first visit (13-25 April 1997) we explored a wide range of habitats from two camp sites: semi-evergreen forest near the headwaters of the Lobéké stream (from 13-21 April), Gilbertiodendron forest near the Boulou (Bolo) stream (21-24 April). Only short visits were paid to Rhynchospora and other swamps, as bird trappers and other poachers made access difficult. A brief (two-day) visit in December 1997 led to the surprising discovery of the Dja River Warbler Bradypterus grandis at one of the Rhynchospora swamps; so a further survey (10-19 April 1999) was organized to investigate the status of this bird further.

Limited bird ringing was undertaken in 1997 ( $180 \mathrm{~m}$ of mist-nets erected during three days in semi-evergreen forest) and 1999 (140 m of mist-nets in Gilbertiodendron forest for three days). Some observations on the Sangha river were made during our visits to Nouabalé-Ndoki N.P. (31 March-20 April 1996 by F.D.L., 25 April-20 May 1997 by both of us). 


\section{Results}

The number of bird species known from Lobéké is 305 (Table 1). We have recorded personally 293 species; other records come mainly from Tim Davenport (who was working in Lobéké in 1997-1998). A full annotated list is given in Dowsett-Lemaire and Dowsett (1999, report deposited at BirdLife International, Cambridge, U.K.); details are given below for only a selection of species of conservation concern. Nomenclature is that of Dowsett and Forbes-Watson (1993).

\section{?Itombwe Nightjar Caprimulgus sp. ?prigoginei}

One in song located c. $1.2 \mathrm{~km}$ east of Boulou camp, singing in the moonlight for more than $20 \mathrm{~min}$ on 21 April 1997, in dense vegetation, some $20 \mathrm{~m}$ high. The song, a dry staccato, was spectrographed and proved identical to that of an unidentified small nightjar taped by F.D.L. near Ndoki camp in 1996 and 1997; the bird also responded to playback of a similar tape of an unidentified nightjar from Itombwe Mt (eastern Zaire, recorded by T. Butynski in 1996). Eventually it flew across the road, sang on the other side, and then much further away (when barely audible). The bird was not re-located in April 1999, and may not have held a territory locally. Now that the song of Brown Nightjar Caprimulgus binotatus is known for certain and is very different (Dowsett-Lemaire and Dowsett 1998a), it is clear this is a third species of forest nightjar, whereas only two are known from central Africa (C. binotatus and the much larger Bates's Nightjar C. batesi). Whilst it may be a new species, the existence of the tape from Itombwe, the locality whence came the single known specimen of Itombwe Nightjar (Louette 1990), suggests that it will probably prove to be that species. It appears much rarer than $C$. binotatus and $C$. batesi, both widespread and locally common in forest in south-east Cameroon and adjacent Congo (pers. obs., see also DowsettLemaire and Dowsett 1998a). In addition to Nouabalé-Ndoki (where three singing birds were located along the 31-km Bomassa-Ndoki track in 1997), we also found it in Nki Faunal Reserve to the west, in two places including the buffer zone at Kupandaka (Dowsett-Lemaire and Dowsett 1998b). Caprimulgus prigoginei is classified as Vulnerable in Collar et al. (1994).

\section{Dja River Warbler Bradypterus grandis}

Classified as Data Deficient by Collar et al. (1994), this is one of the rarest and least known birds in central Africa. Before 1997, it was reported in very small numbers only from two areas in central Gabon (Chaillu and Lopé), and near Bitye in southern Cameroon, where Bates's collector obtained one bird in 1914, the type of the species (Ogilvie-Grant 1917). Thus it was only "rediscovered" in Cameroon in December 1997 when we found at least six singing birds holding territory in Rhynchospora marsh in Lobéké "Petite Savane", in c. 6 ha. Two weeks later, we also found a pair holding a territory in a 1-ha patch of Rhynchospora corymbosa in a small isolated salt-pan (Mala) in Nki Faunal Reserve. The male was mist-netted, ringed and photographed. On our third visit to Lobéké, we found the birds were again very vocal at Petite Savane, but none were singing spontaneously in the much bigger sedge swamps of Djangé marsh. Through per- 
Table 1. Distribution of the birds recorded from three neighbouring protected areas in central Africa. Forest: F, forest species. Chorology is given for Palaearctic migrants (P), and for Afrotropical species with a well-defined affinity to the Guineo-Congolian region (GC). Brackets denote a species not completely endemic to this category. Status (when known): B, breeding visitor; $\mathrm{M}$, migrant (seasonal visitor); N, non-breeding visitor; $\mathrm{R}$, resident; $\mathrm{V}$, vagrant; $\mathrm{W}$, winters. Lobéké: this study; DzangaNdoki, République Centrafricaine: Green and Carroll (1991), Dowsett-Lemaire (* unpubl.); NouabaléNdoki, Congo: Dowsett-Lemaire (1997), Dowsett-Lemaire and Dowsett (1997). For Dzanga-Ndoki National Park we do not include those species reported by Green and Carroll only from villages, savannas or the banks of the Sangha outside the park. For several species noted for the park by these observers, we prefer to await confirmation before accepting them here.

\begin{tabular}{|c|c|c|c|c|c|c|}
\hline Species & Forest & $\begin{array}{l}\text { Choro- } \\
\operatorname{logy}\end{array}$ & Status & Lobéké & $\begin{array}{l}\text { Dzanga- } \\
\text { Ndoki }\end{array}$ & $\begin{array}{l}\text { Nouabalé- } \\
\text { Ndoki }\end{array}$ \\
\hline Little Grebe Tachybaptus ruficollis & & & $\mathrm{N}$ & - & - & $\mathrm{x}$ \\
\hline $\begin{array}{l}\text { Reed Cormorant Phalacrocorax } \\
\text { africanus }\end{array}$ & & & $\mathrm{N}$ & $x$ & $x$ & $x$ \\
\hline Darter Anhinga rufa & & & $\mathrm{N}$ & $x$ & - & $X$ \\
\hline Little Bittern Ixobrychus minutus & & $\mathrm{P}$ & $\mathrm{M}$ & - & - & $\mathrm{X}$ \\
\hline $\begin{array}{l}\text { White-crested Tiger Heron Tigriornis } \\
\text { leucolophus }\end{array}$ & $\mathrm{F}$ & GC & $\mathrm{R}$ & $\mathrm{X}$ & $\mathrm{X}$ & $\mathrm{X}$ \\
\hline $\begin{array}{l}\text { Black-crowned Night Heron } \\
\text { Nycticorax nycticorax }\end{array}$ & & & M? & $\mathrm{x}$ & $\mathrm{x}$ & $X$ \\
\hline $\begin{array}{l}\text { White-backed Night Heron } \\
\text { Gorsachius leuconotus }\end{array}$ & & & $\mathrm{R}$ & $x$ & - & $X$ \\
\hline $\begin{array}{l}\text { Common Squacco Heron Ardeola } \\
\text { ralloides }\end{array}$ & & $\mathrm{P}$ & $\mathrm{W}$ & $x$ & $x$ & $x$ \\
\hline Cattle Egret Bubulcus ibis & & & $\mathrm{N}$ & $\mathrm{x}$ & $\mathrm{x}$ & $\mathrm{x}$ \\
\hline $\begin{array}{l}\text { Green-backed Heron Butorides } \\
\text { striatus }\end{array}$ & & & $\mathrm{R}$ & $x$ & $x$ & $x$ \\
\hline Little Egret Egretta garzetta & & $\mathrm{P}$ & W & $x$ & $X$ & $X$ \\
\hline $\begin{array}{l}\text { Yellow-billed Egret Egretta } \\
\text { intermedia }\end{array}$ & & & $\mathrm{N}$ & $x$ & $X$ & - \\
\hline Great White Egret Egretta alba & & & $\mathrm{M}$ & $\mathrm{X}$ & $\mathrm{X}$ & $\mathrm{X}$ \\
\hline Purple Heron Ardea purpurea & & $\mathrm{P}$ & W & $x$ & $x$ & $X$ \\
\hline Grey Heron Ardea cinerea & & & $\mathrm{N}$ & $x$ & $x$ & $x$ \\
\hline $\begin{array}{l}\text { Black-headed Heron Ardea } \\
\text { melanocephala }\end{array}$ & & & $\mathrm{N}$ & $x$ & $\mathrm{X}$ & $X$ \\
\hline Goliath Heron Ardea goliath & & & V & $x$ & $x$ & - \\
\hline Hamerkop Scopus umbretta & & & $\mathrm{R}$ & $x$ & $x$ & $x$ \\
\hline Abdim's Stork Ciconia abdimii & & & M & $x$ & $x$ & $x$ \\
\hline $\begin{array}{l}\text { Woolly-necked Stork Ciconia } \\
\text { episcopus }\end{array}$ & & & $\mathrm{R}$ & $x$ & $x$ & $x$ \\
\hline $\begin{array}{l}\text { Saddle-billed Stork Ephippiorhynchus } \\
\text { senegalensis }\end{array}$ & & & V & - & $x$ & $x$ \\
\hline $\begin{array}{l}\text { Marabou Stork Leptoptilos } \\
\text { crumeniferus }\end{array}$ & & & V & - & $X$ & $X$ \\
\hline Hadada Bostrychia hagedash & & & $\mathrm{R}$ & $x$ & $x$ & $X$ \\
\hline Green Ibis Bostrychia olivacea & $\mathrm{F}$ & & $\mathrm{R}$ & $x$ & - & $X$ \\
\hline Spot-breasted Ibis Bostrychia rara & $\mathrm{F}$ & $(\mathrm{GC})$ & $\mathrm{R}$ & $x$ & $X^{*}$ & $x$ \\
\hline $\begin{array}{l}\text { Knob-billed Duck Sarkidiornis } \\
\text { melanotos }\end{array}$ & & & M & $x$ & $x$ & $x$ \\
\hline Hartlaub's Duck Pteronetta hartlaubii & F & $(\mathrm{GC})$ & $\mathrm{R}$ & $x$ & $x$ & $X$ \\
\hline Pygmy Goose Nettapus auritus & & & V & $\mathrm{x}$ & - & $x$ \\
\hline Honey Buzzard Pernis apivorus & & $\mathrm{P}$ & & $x$ & - & $X$ \\
\hline Bat Hawk Macheiramphus alcinus & & & $\mathrm{R}$ & - & - & $x$ \\
\hline $\begin{array}{l}\text { Black (Yellow-billed) Kite Milvus } \\
\text { migrans }\end{array}$ & & & M & $x$ & $x$ & $x$ \\
\hline
\end{tabular}


Table 1. cont.

\begin{tabular}{|c|c|c|c|c|c|c|}
\hline Species & Forest & $\begin{array}{l}\text { Choro- } \\
\text { logy }\end{array}$ & Status & Lobéké & $\begin{array}{l}\text { Dzanga- } \\
\text { Ndoki }\end{array}$ & $\begin{array}{l}\text { Nouabalé- } \\
\text { Ndoki }\end{array}$ \\
\hline African Fish Eagle Haliaeetus vocifer & & & $\mathrm{R}$ & $\mathrm{x}$ & $\mathrm{x}$ & $x$ \\
\hline $\begin{array}{l}\text { Palm-nut Vulture Gypohierax } \\
\text { angolensis }\end{array}$ & $\mathrm{F}$ & & $\mathrm{R}$ & $x$ & $x$ & $x$ \\
\hline $\begin{array}{l}\text { Congo Serpent Eagle Dryotriorchis } \\
\text { spectabilis }\end{array}$ & $\mathrm{F}$ & $(\mathrm{GC})$ & $\mathrm{R}$ & $X$ & $x$ & $X$ \\
\hline Gymnogene Polyboroides typus & $\mathrm{F}$ & & $\mathrm{R}$ & $X$ & $\mathrm{X}$ & $X$ \\
\hline $\begin{array}{l}\text { Eurasian Marsh Harrier Circus } \\
\text { aeruginosus }\end{array}$ & & $\mathrm{P}$ & & - & $\mathrm{X}$ & $\mathrm{x}$ \\
\hline $\begin{array}{l}\text { Black Goshawk Accipiter } \\
\text { melanoleucus }\end{array}$ & $\mathrm{F}$ & & $\mathrm{R}$ & $x$ & $x$ & $x$ \\
\hline $\begin{array}{l}\text { Western Little Sparrowhawk } \\
\text { Accipiter erythropus }\end{array}$ & $\mathrm{F}$ & GC & $\mathrm{R}$ & - & $x$ & $X$ \\
\hline $\begin{array}{l}\text { Chestnut-flanked Sparrowhawk } \\
\text { Accipiter castanilius }\end{array}$ & $\mathrm{F}$ & $(\mathrm{GC})$ & $\mathrm{R}$ & $x$ & $x$ & - \\
\hline African Goshawk Accipiter tachiro & $\mathrm{F}$ & & $\mathrm{R}$ & $x$ & - & $x$ \\
\hline $\begin{array}{l}\text { Long-tailed Hawk Urotriorchis } \\
\text { macrourus }\end{array}$ & $\mathrm{F}$ & GC & $\mathrm{R}$ & $x$ & $x$ & $x$ \\
\hline $\begin{array}{l}\text { Lizard Buzzard Kaupifalco } \\
\text { monogrammicus }\end{array}$ & & & $\mathrm{R}$ & - & $X$ & $X$ \\
\hline Common Buzzard Buteo buteo & & $\mathrm{P}$ & M & - & $X^{*}$ & $x$ \\
\hline Red-necked Buzzard Buteo auguralis & & & $\mathrm{M}$ & - & $X$ & $X$ \\
\hline $\begin{array}{l}\text { Lesser Spotted Eagle Aquila } \\
\text { pomarina }\end{array}$ & & $\mathrm{P}$ & M & $x$ & $X^{*}$ & $X$ \\
\hline Booted Eagle Hieraaetus pennatus & & $\mathrm{P}$ & & $X$ & - & - \\
\hline $\begin{array}{l}\text { Ayres's Hawk Eagle Hieraaetus } \\
\text { ayresii }\end{array}$ & $\mathrm{F}$ & & $\mathrm{R}$ & $x$ & - & $x$ \\
\hline $\begin{array}{l}\text { Cassin's Hawk Eagle Spizaetus } \\
\text { africanus }\end{array}$ & $\mathrm{F}$ & $(\mathrm{GC})$ & $\mathrm{R}$ & $x$ & - & $x$ \\
\hline $\begin{array}{l}\text { Long-crested Eagle Lophaetus } \\
\text { occipitalis }\end{array}$ & $\mathrm{F}$ & & $\mathrm{R}$ & $x$ & $x$ & $x$ \\
\hline $\begin{array}{l}\text { Crowned Eagle Stephanoaetus } \\
\text { coronatus }\end{array}$ & $\mathrm{F}$ & & $\mathrm{R}$ & $x$ & $x$ & $x$ \\
\hline Osprey Pandion haliaetus & & $\mathrm{P}$ & $\mathrm{V}$ & $X$ & - & $x$ \\
\hline Lesser Kestrel Falco naumanni & & $\mathrm{P}$ & $\mathrm{V}$ & - & $X^{*}$ & $x$ \\
\hline Peregrine Falcon Falco peregrinus & & $\mathrm{P}$ & & $x$ & - & - \\
\hline Forest Francolin Francolinus lathami & $\mathrm{F}$ & (GC) & $\mathrm{R}$ & $x$ & $x$ & $\mathrm{x}$ \\
\hline $\begin{array}{l}\text { Scaly Francolin Francolinus } \\
\text { squamatus }\end{array}$ & $\mathrm{F}$ & & $\mathrm{R}$ & $\mathrm{X}$ & $\mathrm{X}$ & $\mathrm{X}$ \\
\hline Black Guineafowl Agelastes niger & $\mathrm{F}$ & GC & $\mathrm{R}$ & $X$ & $X$ & $X$ \\
\hline $\begin{array}{l}\text { Plumed Guineafowl Guttera } \\
\text { plumifera }\end{array}$ & $\mathrm{F}$ & GC & $\mathrm{R}$ & $x$ & $X$ & $x$ \\
\hline $\begin{array}{l}\text { Crested Guineafowl Guttera } \\
\text { pucherani }\end{array}$ & $\mathrm{F}$ & & $\mathrm{R}$ & - & $x$ & - \\
\hline $\begin{array}{l}\text { Nkulengu Rail Himantornis } \\
\text { haematopus }\end{array}$ & $\mathrm{F}$ & GC & $\mathrm{R}$ & $X$ & $x$ & $x$ \\
\hline $\begin{array}{l}\text { White-spotted Flufftail Sarothrura } \\
\text { pulchra }\end{array}$ & $\mathrm{F}$ & $(\mathrm{GC})$ & $\mathrm{R}$ & $x$ & $X^{*}$ & $X$ \\
\hline $\begin{array}{l}\text { Buff-spotted Flufftail Sarothrura } \\
\text { elegans }\end{array}$ & $\mathrm{F}$ & & $\mathrm{R}$ & $x$ & - & - \\
\hline Red-chested Flufftail Sarothrura rufa & & & $\mathrm{R}$ & $x$ & - & - \\
\hline Grey-throated Rail Canirallus oculeus & $\mathrm{F}$ & GV & $\mathrm{R}$ & - & - & $x$ \\
\hline African Crake Crecopsis egregia & & & $\mathrm{M}$ & $\mathrm{x}$ & $x$ & - \\
\hline Black Crake Amaurornis flavirostris & & & $\mathrm{R}$ & $\mathrm{X}$ & $x$ & $x$ \\
\hline
\end{tabular}


Table 1. cont.

\begin{tabular}{|c|c|c|c|c|c|c|}
\hline Species & Forest & $\begin{array}{l}\text { Choro- } \\
\operatorname{logy}\end{array}$ & Status & Lobéké & $\begin{array}{l}\text { Dzanga- } \\
\text { Ndoki }\end{array}$ & $\begin{array}{l}\text { Nouabalé- } \\
\text { Ndoki }\end{array}$ \\
\hline African Finfoot Podica senegalensis & $\mathrm{F}$ & & $\mathrm{R}$ & $x$ & $x$ & $x$ \\
\hline African Jacana Actophilornis africanus & & & $\mathrm{R}$ & $\mathrm{X}$ & $\mathrm{X}$ & $\mathrm{X}$ \\
\hline Egyptian Plover Pluvianus aegyptius & & & B & $\mathrm{X}$ & $\mathrm{X}$ & $\mathrm{X}$ \\
\hline $\begin{array}{l}\text { Common Pratincole Glareola } \\
\text { pratincola }\end{array}$ & & & M & - & - & $x$ \\
\hline Rock Pratincole Glareola nuchalis & & & B & $x$ & $\mathrm{X}$ & $\mathrm{X}$ \\
\hline Grey Pratincole Glareola cinerea & & & B & $x$ & $x$ & $x$ \\
\hline $\begin{array}{l}\text { Little Ringed Plover Charadrius } \\
\text { dubius }\end{array}$ & & $\mathrm{P}$ & & $x$ & $x$ & $x$ \\
\hline Ringed Plover Charadrius hiaticula & & $\mathrm{P}$ & & - & $x$ & $x$ \\
\hline Kittlitz's Plover Charadrius pecuarius & & & M & $x$ & - & $\mathrm{X}$ \\
\hline $\begin{array}{l}\text { White-fronted Plover Charadrius } \\
\text { marginatus }\end{array}$ & & & B & $x$ & $X$ & $x$ \\
\hline $\begin{array}{l}\text { White-crowned Plover Vanellus } \\
\text { albiceps }\end{array}$ & & & $\mathrm{R}$ & $x$ & $x$ & $x$ \\
\hline $\begin{array}{l}\text { Brown-chested Wattled Plover } \\
\text { Vanellus superciliosus }\end{array}$ & & & M & - & - & $x$ \\
\hline Common Snipe Gallinago gallinago & & $\mathrm{P}$ & & $x$ & $\mathrm{x}$ & - \\
\hline Great Snipe Gallinago media & & $\mathrm{P}$ & & - & - & $\mathrm{X}$ \\
\hline Marsh Sandpiper Tringa stagnatilis & & $\mathrm{P}$ & & - & $x$ & - \\
\hline Greenshank Tringa nebularia & & $\mathrm{P}$ & W & $x$ & $x$ & $X$ \\
\hline Green Sandpiper Tringa ochropus & & $\mathrm{P}$ & & $x$ & $x$ & $x$ \\
\hline Wood Sandpiper Tringa glareola & & $\mathrm{P}$ & & $x$ & $x$ & $x$ \\
\hline $\begin{array}{l}\text { Common Sandpiper Actitis } \\
\text { hypoleucos }\end{array}$ & & $\mathrm{P}$ & W & $x$ & $x$ & $x$ \\
\hline Little Stint Calidris minuta & & $\mathrm{P}$ & & $\mathrm{x}$ & $\mathrm{x}$ & - \\
\hline Curlew Sandpiper Calidris ferruginea & & $\mathrm{P}$ & & $x$ & $x$ & $\mathrm{X}$ \\
\hline Ruff Philomachus pugnax & & $\mathrm{P}$ & & - & $\mathrm{X}$ & - \\
\hline $\begin{array}{l}\text { African Skimmer Rynchops } \\
\text { flavirostris }\end{array}$ & & & M & $x$ & - & $x$ \\
\hline Afep Pigeon Columba unicincta & F & (GC) & $\mathrm{R}$ & $x$ & $x$ & $X$ \\
\hline $\begin{array}{l}\text { Bronze-naped Pigeon Columba } \\
\text { delegorguei }\end{array}$ & F & & $\mathrm{R}$ & $x$ & $X^{*}$ & $x$ \\
\hline Blue-spotted Dove Turtur afer & $\mathrm{F}$ & & $\mathrm{R}$ & $x$ & $x$ & $x$ \\
\hline $\begin{array}{l}\text { Tambourine Dove Turtur } \\
\text { tympanistria }\end{array}$ & $\mathrm{F}$ & & $\mathrm{R}$ & $x$ & $X^{*}$ & $x$ \\
\hline $\begin{array}{l}\text { Blue-headed Wood Dove Turtur } \\
\text { brehmeri }\end{array}$ & $\mathrm{F}$ & GC & $\mathrm{R}$ & $\mathrm{X}$ & $\mathrm{X}$ & $\mathrm{X}$ \\
\hline Green Pigeon Treron australis & F & & $\mathrm{R}$ & $x$ & $x$ & $x$ \\
\hline $\begin{array}{l}\text { African Grey Parrot Psittacus } \\
\text { erithacus }\end{array}$ & $\mathrm{F}$ & (GC) & $\mathrm{R}$ & $x$ & $x$ & $x$ \\
\hline $\begin{array}{l}\text { Red-fronted Parrot Poicephalus } \\
\text { gulielmi }\end{array}$ & $\mathrm{F}$ & & $\mathrm{R}$ & $x$ & $x$ & $x$ \\
\hline $\begin{array}{l}\text { Red-headed Lovebird Agapornis } \\
\text { pullarius }\end{array}$ & & & $\mathrm{R}$ & - & $x$ & - \\
\hline $\begin{array}{l}\text { Black-collared Lovebird Agapornis } \\
\text { swindernianus }\end{array}$ & $\mathrm{F}$ & GC & $\mathrm{R}$ & $x$ & $x$ & $x$ \\
\hline Guinea Turaco Tauraco persa & F & (GC) & $\mathrm{R}$ & $x$ & $x$ & $x$ \\
\hline $\begin{array}{l}\text { Great Blue Turaco Corythaeola } \\
\text { cristata }\end{array}$ & $\mathrm{F}$ & (GC) & $\mathrm{R}$ & $x$ & $x$ & $x$ \\
\hline Jacobin Cuckoo Clamator jacobinus & & & M & - & - & $x$ \\
\hline Striped Cuckoo Clamator levaillantii & & & $\mathrm{M}$ & - & $\mathrm{X}$ & $\mathrm{X}$ \\
\hline Red-chested Cuckoo Cuculus & F & & $\mathrm{R}$ & $\mathrm{X}$ & $x$ & $\mathrm{X}$ \\
\hline
\end{tabular}


Table 1. cont.

\begin{tabular}{|c|c|c|c|c|c|c|}
\hline Species & Forest & $\begin{array}{l}\text { Choro- } \\
\operatorname{logy}\end{array}$ & Status & Lobéké & $\begin{array}{l}\text { Dzanga- } \\
\text { Ndoki }\end{array}$ & $\begin{array}{l}\text { Nouabalé- } \\
\text { Ndoki }\end{array}$ \\
\hline Black Cuckoo Cuculus clamosus & F & & $\mathrm{R}$ & $x$ & $x$ & $x$ \\
\hline $\begin{array}{l}\text { European Grey Cuckoo Cuculus } \\
\text { canorus }\end{array}$ & & $\mathrm{P}$ & & $x$ & - & - \\
\hline $\begin{array}{l}\text { Dusky Long-tailed Cuckoo } \\
\text { Cercococcyx mechowi }\end{array}$ & F & (GC) & $\mathrm{R}$ & $x$ & $x$ & $x$ \\
\hline $\begin{array}{l}\text { Olive Long-tailed Cuckoo } \\
\text { Cercococcyx olivinus }\end{array}$ & $\mathrm{F}$ & (GC) & $\mathrm{R}$ & $x$ & $X^{*}$ & $x$ \\
\hline $\begin{array}{l}\text { Emerald Cuckoo Chrysococcyx } \\
\text { cupreus }\end{array}$ & $\mathrm{F}$ & & $\mathrm{R}$ & $x$ & $x$ & $x$ \\
\hline $\begin{array}{l}\text { Yellow-throated Green Cuckoo } \\
\text { Chrysococcyx flavigularis }\end{array}$ & $\mathrm{F}$ & GC & $\mathrm{R}$ & $x$ & - & $x$ \\
\hline Klaas's Cuckoo Chrysococcyx klaas & F & & $\mathrm{R}$ & $x$ & $X^{*}$ & $x$ \\
\hline Didric Cuckoo Chrysococcyx caprius & & & $\mathrm{R}$ & $X$ & - & $X$ \\
\hline Green Coucal Ceuthmochares aereus & $\mathrm{F}$ & & $\mathrm{R}$ & $\mathrm{X}$ & $\mathrm{X}$ & $\mathrm{X}$ \\
\hline $\begin{array}{l}\text { African Black Coucal Centropus } \\
\text { grillii }\end{array}$ & & & M & $x$ & - & - \\
\hline Gaboon Coucal Centropus anselli & $\mathrm{F}$ & (GC) & $\mathrm{R}$ & $\mathrm{X}$ & $\mathrm{X}$ & $\mathrm{X}$ \\
\hline $\begin{array}{l}\text { Blue-naped Coucal Centropus } \\
\text { monachus }\end{array}$ & & & $\mathrm{R}$ & $x$ & - & - \\
\hline $\begin{array}{l}\text { Senegal Coucal Centropus } \\
\text { senegalensis }\end{array}$ & & & $\mathrm{R}$ & - & $x$ & - \\
\hline $\begin{array}{l}\text { Sandy Scops Owl Otus } \\
\text { icterorhynchus }\end{array}$ & F & GC & $\mathrm{R}$ & $x$ & $X^{*}$ & $x$ \\
\hline Fraser's Eagle Owl Bubo poensis & $\mathrm{F}$ & & $\mathrm{R}$ & $x$ & $\mathrm{X}$ & $\mathrm{X}$ \\
\hline Akun Eagle Owl Bubo leucostictus & F & GC & $\mathrm{R}$ & $x$ & - & $X$ \\
\hline Pel's Fishing Owl Scotopelia peli & F & & $\mathrm{R}$ & $x$ & $X^{*}$ & $x$ \\
\hline $\begin{array}{l}\text { Bouvier's Fishing Owl Scotopelia } \\
\text { bouvieri }\end{array}$ & $\mathrm{F}$ & GC & $\mathrm{R}$ & $x$ & $X^{*}$ & $x$ \\
\hline $\begin{array}{l}\text { Red-chested Owlet Glaucidium } \\
\text { tephronotum }\end{array}$ & F & (GC) & $\mathrm{R}$ & $x$ & - & $x$ \\
\hline Barred Owlet Glaucidium capense & $\mathrm{F}$ & & $\mathrm{R}$ & $x$ & - & $\mathrm{X}$ \\
\hline Wood Owl Strix woodfordii & $\mathrm{F}$ & & $\mathrm{R}$ & $\mathrm{X}$ & $\mathrm{X}$ & $\mathrm{X}$ \\
\hline $\begin{array}{l}\text { Brown Nightjar Caprimulgus } \\
\text { binotatus }\end{array}$ & $\mathrm{F}$ & GC & $\mathrm{R}$ & $x$ & $x$ & $X$ \\
\hline Bates's Nightjar Caprimulgus batesi & F & GC & $\mathrm{R}$ & $x$ & - & $X$ \\
\hline $\begin{array}{l}\text { Unident. Nightjar Caprimulgus sp. } \\
\text { (prigoginei?) }\end{array}$ & $\mathrm{F}$ & GC & $\mathrm{R}$ & $x$ & - & $x$ \\
\hline $\begin{array}{l}\text { Pennant-winged Nightjar } \\
\text { Macrodipteryx vexillarius }\end{array}$ & & & M & $x$ & $x$ & $x$ \\
\hline $\begin{array}{l}\text { Chapin's Spinetail Telacanthura } \\
\text { melanopygia }\end{array}$ & $\mathrm{F}$ & GC & $\mathrm{R}$ & $x$ & $x$ & $X$ \\
\hline Sabine's Spinetail Rhaphidura sabini & F & (GC) & $\mathrm{R}$ & $x$ & $x$ & $x$ \\
\hline Cassin's Spinetail Neafrapus cassini & $\mathrm{F}$ & GC & $\mathrm{R}$ & $\mathrm{X}$ & $\mathrm{X}$ & $\mathrm{X}$ \\
\hline African Palm Swift Cypsiurus parous & & & $\mathrm{R}$ & $X$ & $x$ & $X$ \\
\hline European Swift Apus apus & & $\mathrm{P}$ & & $x$ & $X^{*}$ & $\mathrm{X}$ \\
\hline Little Swift Apus affinis & & & $\mathrm{R}$ & - & $\mathrm{X}$ & $\mathrm{X}$ \\
\hline Horus Swift Apus horus & & & $\mathrm{R}$ & - & - & $\mathrm{X}$ \\
\hline Bates's Swift Apus batesi & F & GC & $\mathrm{R}$ & $x$ & - & $x$ \\
\hline Narina Trogon Apaloderma narina & F & & $\mathrm{R}$ & $x$ & $x$ & $X$ \\
\hline $\begin{array}{l}\text { Bare-cheeked Trogon Apaloderma } \\
\text { aequatoriale }\end{array}$ & $\mathrm{F}$ & GC & $\mathrm{R}$ & $x$ & $X^{*}$ & $x$ \\
\hline Shining-blue Kingfisher Alcedo & F & (GC) & $\mathrm{R}$ & $x$ & $x$ & $x$ \\
\hline
\end{tabular}


Table 1. cont.

\begin{tabular}{|c|c|c|c|c|c|c|}
\hline Species & Forest & $\begin{array}{l}\text { Choro- } \\
\log y\end{array}$ & Status & Lobéké & $\begin{array}{l}\text { Dzanga- } \\
\text { Ndoki }\end{array}$ & $\begin{array}{l}\text { Nouabalé- } \\
\text { Ndoki }\end{array}$ \\
\hline Malachite Kingfisher Alcedo cristata & & & $\mathrm{R}$ & $\mathrm{x}$ & $\mathrm{x}$ & $\mathrm{x}$ \\
\hline $\begin{array}{l}\text { White-bellied Kingfisher Alcedo } \\
\text { leucogaster }\end{array}$ & $\mathrm{F}$ & $(\mathrm{GC})$ & $\mathrm{R}$ & $x$ & $\mathrm{x}$ & $x$ \\
\hline Dwarf Kingfisher Ceyx lecontei & F & (GC) & $\mathrm{R}$ & $\mathrm{X}$ & $x$ & $X$ \\
\hline Pygmy Kingfisher Ceyx pictus & & & $\mathrm{R}$ & $\mathrm{X}$ & $\mathrm{X}$ & $\mathrm{X}$ \\
\hline $\begin{array}{l}\text { Chocolate-backed Kingfisher } \\
\text { Halcyon badia }\end{array}$ & $\mathrm{F}$ & GC & $\mathrm{R}$ & $x$ & $X$ & $\mathrm{X}$ \\
\hline $\begin{array}{l}\text { Chestnut-bellied Kingfisher Halcyon } \\
\text { leucocephala }\end{array}$ & & & M & $x$ & - & - \\
\hline $\begin{array}{l}\text { Blue-breasted Kingfisher Halcyon } \\
\text { malimbica }\end{array}$ & $\mathrm{F}$ & & $\mathrm{R}$ & $x$ & $X^{*}$ & $X$ \\
\hline $\begin{array}{l}\text { Senegal Kingfisher Halcyon } \\
\text { senegalensis }\end{array}$ & & & $\mathrm{R}$ & $x$ & $X$ & $X$ \\
\hline Giant Kingfisher Megaceryle maxima & & & $\mathrm{R}$ & $\mathrm{x}$ & $\mathrm{x}$ & $\mathrm{X}$ \\
\hline Pied Kingfisher Ceryle rudis & & & $\mathrm{R}$ & $\mathrm{x}$ & $X$ & $x$ \\
\hline $\begin{array}{l}\text { Black-headed Bee-eater Merops } \\
\text { breweri }\end{array}$ & $\mathrm{F}$ & $(\mathrm{GC})$ & $\mathrm{R}$ & - & $X$ & - \\
\hline $\begin{array}{l}\text { Blue-headed Bee-eater Merops } \\
\text { muelleri }\end{array}$ & $\mathrm{F}$ & $(\mathrm{GC})$ & $\mathrm{R}$ & $x$ & $X$ & $X$ \\
\hline Black Bee-eater Merops gularis & $\mathrm{F}$ & $(\mathrm{GC})$ & $\mathrm{R}$ & $X$ & $x$ & $X$ \\
\hline $\begin{array}{l}\text { White-cheeked Bee-eater Merops } \\
\text { variegatus }\end{array}$ & & & $\mathrm{R}$ & $X$ & $X$ & $X$ \\
\hline $\begin{array}{l}\text { White-throated Bee-eater Merops } \\
\text { albicollis }\end{array}$ & & & M & $x$ & $\mathrm{x}$ & $X$ \\
\hline European Bee-eater Merops apiaster & & $\mathrm{P}$ & M & $x$ & - & $x$ \\
\hline $\begin{array}{l}\text { Blue-throated Roller Eurystomus } \\
\text { gularis }\end{array}$ & $\mathrm{F}$ & $(\mathrm{GC})$ & $\mathrm{R}$ & $\mathrm{x}$ & $\mathrm{X}$ & $X$ \\
\hline $\begin{array}{l}\text { White-headed Wood Hoopoe } \\
\text { Phoeniculus bollei }\end{array}$ & $\mathrm{F}$ & & $\mathrm{R}$ & $x$ & $X$ & - \\
\hline $\begin{array}{l}\text { Forest Wood Hoopoe Phoeniculus } \\
\text { castaneiceps }\end{array}$ & $\mathrm{F}$ & (GC) & $\mathrm{R}$ & $x$ & - & $X$ \\
\hline $\begin{array}{l}\text { White-crested Hornbill Tropicranus } \\
\text { albocristatus }\end{array}$ & $\mathrm{F}$ & (GC) & $\mathrm{R}$ & $x$ & $X$ & $X$ \\
\hline $\begin{array}{l}\text { Black Dwarf Hornbill Tockus } \\
\text { hartlaubi }\end{array}$ & $\mathrm{F}$ & GC & $\mathrm{R}$ & $\mathrm{x}$ & $\mathrm{X}$ & $\mathrm{X}$ \\
\hline $\begin{array}{l}\text { Red-billed Dwarf Hornbill Tockus } \\
\text { camurus }\end{array}$ & $\mathrm{F}$ & $(\mathrm{GC})$ & $\mathrm{R}$ & $x$ & $x$ & $x$ \\
\hline Pied Hornbill Tockus fasciatus & $\mathrm{F}$ & (GC) & $\mathrm{R}$ & $x$ & $x$ & $X$ \\
\hline $\begin{array}{l}\text { White-tailed Hornbill Bycanistes } \\
\text { fistulator }\end{array}$ & $\mathrm{F}$ & (GC) & $\mathrm{R}$ & $x$ & $X$ & $X$ \\
\hline $\begin{array}{l}\text { White-thighed Hornbill Bycanistes } \\
\text { cylindricus }\end{array}$ & $\mathrm{F}$ & (GC) & $\mathrm{R}$ & $x$ & $x$ & $X$ \\
\hline $\begin{array}{l}\text { Black-and-white Casqued Hornbill } \\
\text { Bycanistes subcylindricus }\end{array}$ & $\mathrm{F}$ & $(\mathrm{GC})$ & $\mathrm{R}$ & $x$ & $x$ & $x$ \\
\hline $\begin{array}{l}\text { Black-wattled Hornbill Ceratogymna } \\
\text { atrata }\end{array}$ & $\mathrm{F}$ & (GC) & $\mathrm{R}$ & $x$ & $X$ & $X$ \\
\hline $\begin{array}{l}\text { Grey-throated Barbet Gymnobucco } \\
\text { bonapartei }\end{array}$ & $\mathrm{F}$ & & $\mathrm{R}$ & $\mathrm{x}$ & $\mathrm{X}$ & $\mathrm{X}$ \\
\hline $\begin{array}{l}\text { Yellow-spotted Barbet Buccanodon } \\
\text { duchaillui }\end{array}$ & $\mathrm{F}$ & $(\mathrm{GC})$ & $\mathrm{R}$ & $x$ & $x$ & $x$ \\
\hline $\begin{array}{l}\text { Speckled Tinkerbird Pogoniulus } \\
\text { scolopaceus }\end{array}$ & $\mathrm{F}$ & (GC) & $\mathrm{R}$ & $X$ & $x$ & $X$ \\
\hline $\begin{array}{l}\text { Golden-rumped Tinkerbird } \\
\text { Pogoniulus bilineatus }\end{array}$ & $\mathrm{F}$ & & $\mathrm{R}$ & $\mathrm{x}$ & $X$ & $\mathrm{X}$ \\
\hline
\end{tabular}


Table 1. cont.

\begin{tabular}{|c|c|c|c|c|c|c|}
\hline Species & Forest & $\begin{array}{l}\text { Choro- } \\
\log y\end{array}$ & Status & Lobéké & $\begin{array}{l}\text { Dzanga- } \\
\text { Ndoki }\end{array}$ & $\begin{array}{l}\text { Nouabalé- } \\
\text { Ndoki }\end{array}$ \\
\hline $\begin{array}{l}\text { Yellow-throated Tinkerbird } \\
\text { Pogoniulus subsulphureus }\end{array}$ & $\mathrm{F}$ & $(\mathrm{GC})$ & $\mathrm{R}$ & $x$ & $X^{*}$ & $x$ \\
\hline $\begin{array}{l}\text { Red-rumped Tinkerbird Pogoniulus } \\
\text { atroflavus }\end{array}$ & F & $(\mathrm{GC})$ & $\mathrm{R}$ & $x$ & $x$ & $x$ \\
\hline $\begin{array}{l}\text { Hairy-breasted Barbet Tricholaema } \\
\text { hirsuta }\end{array}$ & F & (GC) & $\mathrm{R}$ & $x$ & $X$ & $X$ \\
\hline $\begin{array}{l}\text { Yellow-billed Barbet Trachyphonus } \\
\text { purpuratus }\end{array}$ & $\mathrm{F}$ & (GC) & $\mathrm{R}$ & $x$ & $x$ & $x$ \\
\hline $\begin{array}{l}\text { Cassin's Honeyguide Prodotiscus } \\
\text { insignis }\end{array}$ & F & $(\mathrm{GC})$ & $\mathrm{R}$ & - & - & $x$ \\
\hline $\begin{array}{l}\text { Zenker's Honeyguide Melignomon } \\
\text { zenkeri }\end{array}$ & F & GC & $\mathrm{R}$ & $x$ & - & $x$ \\
\hline $\begin{array}{l}\text { Spotted Honeyguide Indicator } \\
\text { maculatus }\end{array}$ & F & $(\mathrm{GC})$ & $\mathrm{R}$ & $x$ & - & $X$ \\
\hline Lesser Honeyguide Indicator minor & $\mathrm{F}$ & & $\mathrm{R}$ & $\mathrm{x}$ & - & $\mathrm{X}$ \\
\hline $\begin{array}{l}\text { Western Least Honeyguide Indicator } \\
\text { exilis }\end{array}$ & $\mathrm{F}$ & $(\mathrm{GC})$ & $\mathrm{R}$ & $x$ & - & $x$ \\
\hline $\begin{array}{l}\text { Willcocks's Honeyguide Indicator } \\
\text { willcocksi }\end{array}$ & F & (GC) & $\mathrm{R}$ & $x$ & $X^{*}$ & $X$ \\
\hline $\begin{array}{l}\text { Lyre-tailed Honeyguide Melichneutes } \\
\text { robustus }\end{array}$ & F & GC & $\mathrm{R}$ & $x$ & $x$ & $x$ \\
\hline Piculet Sasia africana & $\mathrm{F}$ & (GC) & $\mathrm{R}$ & $x$ & $\mathrm{X}$ & $\mathrm{X}$ \\
\hline $\begin{array}{l}\text { Little Spotted Woodpecker } \\
\text { Campethera cailliautii }\end{array}$ & F & & $\mathrm{R}$ & $X$ & $X$ & $x$ \\
\hline $\begin{array}{l}\text { Buff-spotted Woodpecker } \\
\text { Campethera nivosa }\end{array}$ & F & $(\mathrm{GC})$ & $\mathrm{R}$ & $x$ & $X^{*}$ & $x$ \\
\hline $\begin{array}{l}\text { Brown-eared Woodpecker } \\
\text { Campethera caroli }\end{array}$ & F & $(\mathrm{GC})$ & $\mathrm{R}$ & $x$ & $x$ & $X$ \\
\hline $\begin{array}{l}\text { Gabon Woodpecker Dendropicos } \\
\text { gabonensis }\end{array}$ & F & GC & $\mathrm{R}$ & $x$ & $x$ & $x$ \\
\hline $\begin{array}{l}\text { Yellow-crested Woodpecker Thripias } \\
\text { xantholophus }\end{array}$ & F & $(\mathrm{GC})$ & $\mathrm{R}$ & $x$ & $x$ & $x$ \\
\hline Elliot's Woodpecker Mesopicos elliotii & $\mathrm{F}$ & $(\mathrm{GC})$ & $\mathrm{R}$ & $x$ & $x$ & $x$ \\
\hline $\begin{array}{l}\text { Rufous-sided Broadbill Smithornis } \\
\text { rufolateralis }\end{array}$ & $\mathrm{F}$ & GC & $\mathrm{R}$ & $\mathrm{x}$ & $X^{*}$ & $\mathrm{X}$ \\
\hline $\begin{array}{l}\text { Square-tailed Saw-wing } \\
\text { Psalidoprocne nitens }\end{array}$ & $\mathrm{F}$ & GC & $\mathrm{R}$ & $x$ & $x$ & $x$ \\
\hline $\begin{array}{l}\text { Black Saw-wing Psalidoprocne } \\
\text { pristoptera }\end{array}$ & & & $\mathrm{R}$ & - & $x$ & - \\
\hline $\begin{array}{l}\text { Red-breasted Swallow Hirundo } \\
\text { semirufa }\end{array}$ & & & $\mathrm{R}$ & - & $x$ & $x$ \\
\hline $\begin{array}{l}\text { Mosque Swallow Hirundo } \\
\text { senegalensis }\end{array}$ & & & $\mathrm{R}$ & - & $x$ & $x$ \\
\hline $\begin{array}{l}\text { Lesser Striped Swallow Hirundo } \\
\text { abyssinica }\end{array}$ & & & $\mathrm{R}$ & - & - & $X$ \\
\hline Wire-tailed Swallow Hirundo smithii & & & $\mathrm{R}$ & - & - & $x$ \\
\hline $\begin{array}{l}\text { White-throated Blue Swallow } \\
\text { Hirundo nigrita }\end{array}$ & $\mathrm{F}$ & $(\mathrm{GC})$ & $\mathrm{R}$ & $x$ & $x$ & $x$ \\
\hline European Swallow Hirundo rustica & & $\mathrm{P}$ & W & $x$ & $\mathrm{x}$ & $x$ \\
\hline House Martin Delichon urbica & & $\mathrm{P}$ & & $x$ & - & - \\
\hline Yellow Wagtail Motacilla flava & & $\mathrm{P}$ & W & $x$ & $x$ & $\mathrm{X}$ \\
\hline Mountain Wagtail Motacilla clara & & & $\mathrm{R}$ & $x$ & - & $x$ \\
\hline Purple-throated Cuckoo-shrike & $\mathrm{F}$ & & $\mathrm{R}$ & $\mathrm{x}$ & $x$ & $\mathrm{X}$ \\
\hline
\end{tabular}


Table 1. cont.

\begin{tabular}{|c|c|c|c|c|c|c|}
\hline Species & Forest & $\begin{array}{l}\text { Choro- } \\
\text { logy }\end{array}$ & Status & Lobéké & $\begin{array}{l}\text { Dzanga- } \\
\text { Ndoki }\end{array}$ & $\begin{array}{l}\text { Nouabalé- } \\
\text { Ndoki }\end{array}$ \\
\hline Blue Cuckoo-shrike Coracina azurea & $\mathrm{F}$ & GC & $\mathrm{R}$ & $\mathrm{x}$ & $\mathrm{x}$ & $x$ \\
\hline Little Greenbul Andropadus virens & $\mathrm{F}$ & & $\mathrm{R}$ & $x$ & $x$ & $x$ \\
\hline $\begin{array}{l}\text { Little Grey Greenbul Andropadus } \\
\text { gracilis }\end{array}$ & $\mathrm{F}$ & (GC) & $\mathrm{R}$ & $x$ & $X^{*}$ & $x$ \\
\hline $\begin{array}{l}\text { Ansorge's Greenbul Andropadus } \\
\text { ansorgei }\end{array}$ & $\mathrm{F}$ & (GC) & $\mathrm{R}$ & $X$ & $X^{*}$ & $\mathrm{x}$ \\
\hline $\begin{array}{l}\text { Cameroon Sombre Greenbul } \\
\text { Andropadus curvirostris }\end{array}$ & $\mathrm{F}$ & (GC) & $\mathrm{R}$ & $\mathrm{X}$ & $\mathrm{X}^{*}$ & $\mathrm{x}$ \\
\hline $\begin{array}{l}\text { Slender-billed Greenbul Andropadus } \\
\text { gracilirostris }\end{array}$ & $\mathrm{F}$ & & $\mathrm{R}$ & $x$ & $X^{*}$ & $x$ \\
\hline $\begin{array}{l}\text { Yellow-whiskered Greenbul } \\
\text { Andropadus latirostris }\end{array}$ & $\mathrm{F}$ & & & $x$ & $X^{*}$ & $x$ \\
\hline Golden Greenbul Calyptocichla serina & $\mathrm{F}$ & GC & $\mathrm{R}$ & $X$ & $X^{*}$ & $X$ \\
\hline $\begin{array}{l}\text { Honeyguide Greenbul Baeopogon } \\
\text { indicator }\end{array}$ & $\mathrm{F}$ & (GC) & $\mathrm{R}$ & $x$ & $X^{*}$ & $x$ \\
\hline $\begin{array}{l}\text { Sjöstedt's Honeyguide Greenbul } \\
\text { Baeopogon clamans }\end{array}$ & $\mathrm{F}$ & GC & $\mathrm{R}$ & $x$ & $X^{*}$ & $x$ \\
\hline Spotted Greenbul Ixonotus guttatus & $\mathrm{F}$ & (GC) & $\mathrm{R}$ & $\mathrm{X}$ & $x$ & $x$ \\
\hline Simple Greenbul Chlorocichla simplex & $\mathrm{F}$ & (GC) & $\mathrm{R}$ & $X$ & - & $X$ \\
\hline $\begin{array}{l}\text { Yellow-throated Leaflove } \\
\text { Chlorocichla flavicollis }\end{array}$ & $\mathrm{F}$ & & $\mathrm{R}$ & $\mathrm{X}$ & $\mathrm{X}$ & $\mathrm{X}$ \\
\hline $\begin{array}{l}\text { Swamp Palm Bulbul Thescelocichla } \\
\text { leucopleura }\end{array}$ & $\mathrm{F}$ & (GC) & $\mathrm{R}$ & $x$ & $x$ & $x$ \\
\hline Leaflove Pyrrhurus scandens & $\mathrm{F}$ & (GC) & $\mathrm{R}$ & - & $X$ & $X$ \\
\hline $\begin{array}{l}\text { Icterine Greenbul Phyllastrephus } \\
\text { icterinus }\end{array}$ & $\mathrm{F}$ & (GC) & $\mathrm{R}$ & $X$ & $X^{*}$ & $x$ \\
\hline $\begin{array}{l}\text { Xavier's Greenbul Phyllastrephus } \\
\quad \text { xavieri }\end{array}$ & $\mathrm{F}$ & (GC) & $\mathrm{R}$ & $x$ & $X^{*}$ & $x$ \\
\hline $\begin{array}{l}\text { White-throated Greenbul } \\
\text { Phyllastrephus albigularis }\end{array}$ & $\mathrm{F}$ & (GC) & $\mathrm{R}$ & $x$ & $X^{*}$ & $x$ \\
\hline Bristlebill Bleda syndactyla & $\mathrm{F}$ & (GC) & $\mathrm{R}$ & $\mathrm{X}$ & $x$ & $x$ \\
\hline $\begin{array}{l}\text { E. Green-tailed Bristlebill Bleda } \\
\text { (eximia) notata }\end{array}$ & $\mathrm{F}$ & GC & $\mathrm{R}$ & $X$ & $X^{*}$ & $X$ \\
\hline $\begin{array}{l}\text { Eastern Bearded Greenbul Criniger } \\
\text { chloronotus }\end{array}$ & $\mathrm{F}$ & GC & $\mathrm{R}$ & $x$ & $X^{*}$ & $x$ \\
\hline Red-tailed Greenbul Criniger calurus & $\mathrm{F}$ & GC & $\mathrm{R}$ & $x$ & $\mathrm{X}$ & $x$ \\
\hline $\begin{array}{l}\text { White-bearded Greenbul Criniger } \\
\text { olivaceus }\end{array}$ & $\mathrm{F}$ & GC & $\mathrm{R}$ & $\mathrm{X}$ & $\mathrm{X}^{*}$ & $\mathrm{X}$ \\
\hline Common Bulbul Pycnonotus barbatus & & & $\mathrm{R}$ & $X$ & $X$ & $X$ \\
\hline $\begin{array}{l}\text { Red-tailed Ant Thrush Neocossyphus } \\
\text { rufus }\end{array}$ & $\mathrm{F}$ & & $\mathrm{R}$ & $x$ & $X^{*}$ & $x$ \\
\hline $\begin{array}{l}\text { White-tailed Ant Thrush } \\
\text { Neocossyphus poensis }\end{array}$ & $\mathrm{F}$ & (GC) & $\mathrm{R}$ & $x$ & - & $x$ \\
\hline Rufous Ant Thrush Stizorhina fraseri & $\mathrm{F}$ & (GC) & $\mathrm{R}$ & $\mathrm{X}$ & $X^{*}$ & $x$ \\
\hline Fire-crested Alethe Alethe diademata & $\mathrm{F}$ & (GC) & $\mathrm{R}$ & $X$ & $X^{*}$ & $X$ \\
\hline $\begin{array}{l}\text { Brown-chested Alethe Alethe } \\
\text { poliocephala }\end{array}$ & $\mathrm{F}$ & & $\mathrm{R}$ & $x$ & $X^{*}$ & $x$ \\
\hline $\begin{array}{l}\text { Forest Robin Stiphrornis } \\
\text { erythrothorax }\end{array}$ & $\mathrm{F}$ & (GC) & $\mathrm{R}$ & $x$ & $X^{*}$ & $x$ \\
\hline Akalat Sheppardia cyornithopsis & $\mathrm{F}$ & (GC) & $\mathrm{R}$ & $x$ & $X^{*}$ & $x$ \\
\hline $\begin{array}{l}\text { Blue-shouldered Robin Cossypha } \\
\text { cyanocampter }\end{array}$ & $\mathrm{F}$ & (GC) & $\mathrm{R}$ & $x$ & $X^{*}$ & $x$ \\
\hline Whinchat Saxicola rubetra & & $\mathrm{P}$ & W & $\mathrm{x}$ & - & - \\
\hline
\end{tabular}


Table 1. cont.

\begin{tabular}{|c|c|c|c|c|c|c|}
\hline Species & Forest & $\begin{array}{l}\text { Choro- } \\
\text { logy }\end{array}$ & Status & Lobéké & $\begin{array}{l}\text { Dzanga- } \\
\text { Ndoki }\end{array}$ & $\begin{array}{l}\text { Nouabalé- } \\
\text { Ndoki }\end{array}$ \\
\hline Dja River Warbler Bradypterus grandis & & GC & $\mathrm{R}$ & $\mathrm{X}$ & - & - \\
\hline $\begin{array}{l}\text { Black-faced Rufous Warbler } \\
\text { Bathmocercus rufus }\end{array}$ & $\mathrm{F}$ & (GC) & $\mathrm{R}$ & $X$ & $\mathrm{X}$ & $\mathrm{X}$ \\
\hline $\begin{array}{l}\text { Sedge Warbler Acrocephalus } \\
\text { schoenobaenus }\end{array}$ & & $\mathrm{P}$ & & $X$ & $X$ & $X$ \\
\hline Reed Warbler Acrocephalus scirpaceus & & $\mathrm{P}$ & W & $x$ & - & - \\
\hline $\begin{array}{l}\text { Great Reed Warbler Acrocephalus } \\
\text { arundinaceus }\end{array}$ & & $\mathrm{P}$ & W & $x$ & - & - \\
\hline $\begin{array}{l}\text { Rufous-crowned Eremomela } \\
\text { Eremomela badiceps }\end{array}$ & $\mathrm{F}$ & (GC) & $\mathrm{R}$ & $x$ & - & $x$ \\
\hline Green Crombec Sylvietta virens & $\mathrm{F}$ & (GC) & $\mathrm{R}$ & $X$ & $\mathrm{X}$ & $\mathrm{X}$ \\
\hline $\begin{array}{l}\text { Lemon-bellied Crombec Sylvietta } \\
\text { denti }\end{array}$ & $\mathrm{F}$ & GC & $\mathrm{R}$ & $x$ & $X^{*}$ & $x$ \\
\hline Grey Longbill Macrosphenus concolor & $\mathrm{F}$ & (GC) & $\mathrm{R}$ & $x$ & $X^{*}$ & $x$ \\
\hline $\begin{array}{l}\text { Yellow Longbill Macrosphenus } \\
\text { flavicans }\end{array}$ & F & (GC) & $\mathrm{R}$ & $X$ & $X^{*}$ & $X$ \\
\hline $\begin{array}{l}\text { Willow Warbler Phylloscopus } \\
\text { trochilus }\end{array}$ & & $\mathrm{P}$ & M & - & - & $X$ \\
\hline $\begin{array}{l}\text { Uganda Woodland Warbler } \\
\text { Phylloscopus budongoensis }\end{array}$ & F & $(\mathrm{GC})$ & $\mathrm{R}$ & $X$ & - & - \\
\hline $\begin{array}{l}\text { Violet-backed Hyliota Hyliota } \\
\text { violacea }\end{array}$ & $\mathrm{F}$ & GC & $\mathrm{R}$ & $x$ & - & $x$ \\
\hline Green Hylia Hylia prasina & $\mathrm{F}$ & (GC) & $\mathrm{R}$ & $x$ & $X^{*}$ & $X$ \\
\hline $\begin{array}{l}\text { Chattering Cisticola Cisticola } \\
\text { anonymus }\end{array}$ & & (GC) & $\mathrm{R}$ & $x$ & 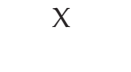 & $X$ \\
\hline $\begin{array}{l}\text { White-chinned Prinia Prinia } \\
\text { leucopogon }\end{array}$ & F & (GC) & $\mathrm{R}$ & - & - & $X$ \\
\hline Banded Prinia Prinia bairdii & $\mathrm{F}$ & & $\mathrm{R}$ & $x$ & $x$ & $X$ \\
\hline Black-throated Apalis Apalis jacksoni & $\mathrm{F}$ & & $\mathrm{R}$ & $\mathrm{X}$ & - & - \\
\hline Black-capped Apalis Apalis nigriceps & $\mathrm{F}$ & (GC) & $\mathrm{R}$ & $\mathrm{X}$ & - & - \\
\hline $\begin{array}{l}\text { Buff-throated Apalis Apalis } \\
\text { rufogularis }\end{array}$ & $\mathrm{F}$ & (GC) & $\mathrm{R}$ & $X$ & $X^{*}$ & $X$ \\
\hline Gosling's Apalis Apalis goslingi & $\mathrm{F}$ & (GC) & $\mathrm{R}$ & $\mathrm{X}$ & $\mathrm{X}^{*}$ & $\mathrm{X}$ \\
\hline $\begin{array}{l}\text { Bleating Bush Warbler Camaroptera } \\
\text { brachyura }\end{array}$ & & & $\mathrm{R}$ & $X$ & $x$ & $X$ \\
\hline $\begin{array}{l}\text { Yellow-browed Camaroptera } \\
\text { Camaroptera superciliaris }\end{array}$ & $\mathrm{F}$ & (GC) & $\mathrm{R}$ & $\mathrm{x}$ & $\mathrm{X}^{*}$ & $\mathrm{X}$ \\
\hline $\begin{array}{l}\text { Olive-green Camaroptera } \\
\text { Camaroptera chloronota }\end{array}$ & $\mathrm{F}$ & (GC) & $\mathrm{R}$ & $x$ & $X^{*}$ & $X$ \\
\hline Forest Flycatcher Fraseria ocreata & $\mathrm{F}$ & GC & $\mathrm{R}$ & $x$ & $x$ & $X$ \\
\hline $\begin{array}{l}\text { White-browed Forest Flycatcher } \\
\text { Fraseria cinerascens }\end{array}$ & $\mathrm{F}$ & GC & $\mathrm{R}$ & $X$ & $X^{*}$ & $X$ \\
\hline Spotted Flycatcher Muscicapa striata & & $\mathrm{P}$ & M & - & $x$ & - \\
\hline $\begin{array}{l}\text { Olivaceous Flycatcher Muscicapa } \\
\text { olivascens }\end{array}$ & $\mathrm{F}$ & GC & $\mathrm{R}$ & $\mathrm{X}$ & $\mathrm{X}$ & $\mathrm{X}$ \\
\hline $\begin{array}{l}\text { Cassin's Grey Flycatcher Muscicapa } \\
\text { cassini }\end{array}$ & $\mathrm{F}$ & (GC) & $\mathrm{R}$ & $X$ & $X$ & $X$ \\
\hline $\begin{array}{l}\text { Little Grey Flycatcher Muscicapa } \\
\text { epulata }\end{array}$ & $\mathrm{F}$ & GC & $\mathrm{R}$ & $X$ & - & - \\
\hline $\begin{array}{l}\text { Yellow-footed Flycatcher Muscicapa } \\
\text { sethsmithi }\end{array}$ & $\mathrm{F}$ & GC & $\mathrm{R}$ & $X$ & $X$ & $X$ \\
\hline Ashy Flycatcher Muscicapa & $\mathrm{F}$ & & $\mathrm{R}$ & $x$ & - & $x$ \\
\hline
\end{tabular}


Table 1. cont.

\begin{tabular}{|c|c|c|c|c|c|c|}
\hline Species & Forest & $\begin{array}{l}\text { Choro- } \\
\operatorname{logy}\end{array}$ & Status & Lobéké & $\begin{array}{l}\text { Dzanga- } \\
\text { Ndoki }\end{array}$ & $\begin{array}{l}\text { Nouabalé- } \\
\text { Ndoki }\end{array}$ \\
\hline $\begin{array}{l}\text { Dusky Blue Flycatcher Muscicapa } \\
\text { comitata }\end{array}$ & $\mathrm{F}$ & (GC) & $\mathrm{R}$ & $x$ & $x$ & $x$ \\
\hline Sooty Flycatcher Muscicapa infuscata & $\mathrm{F}$ & (GC) & $\mathrm{R}$ & $x$ & $x$ & $x$ \\
\hline $\begin{array}{l}\text { Tessmann's Flycatcher Muscicapa } \\
\text { tessmanni }\end{array}$ & $\mathrm{F}$ & GC & $\mathrm{R}$ & $x$ & - & - \\
\hline $\begin{array}{l}\text { Lead-coloured Flycatcher Myioparus } \\
\text { plumbeus }\end{array}$ & & & $\mathrm{R}$ & $x$ & $x$ & $x$ \\
\hline $\begin{array}{l}\text { Grey-throated Flycatcher Myioparus } \\
\text { griseigularis }\end{array}$ & $\mathrm{F}$ & (GC) & $\mathrm{R}$ & $x$ & $X^{*}$ & $X$ \\
\hline $\begin{array}{l}\text { Shrike-Flycatcher Megabyas } \\
\text { flammulatus }\end{array}$ & $\mathrm{F}$ & (GC) & $\mathrm{R}$ & $X$ & - & $X$ \\
\hline $\begin{array}{l}\text { Black-and-white Flycatcher Bias } \\
\text { musicus }\end{array}$ & F & & $\mathrm{R}$ & - & $X$ & $X$ \\
\hline Fernando Po Batis Batis poensis & $\mathrm{F}$ & GC & $\mathrm{R}$ & $x$ & $X^{*}$ & $X$ \\
\hline $\begin{array}{l}\text { Yellow-bellied Wattle-eye } \\
\text { Dyaphorophyia concreta }\end{array}$ & F & (GC) & $\mathrm{R}$ & - & $X$ & $X$ \\
\hline $\begin{array}{l}\text { Black-necked Wattle-eye } \\
\text { Dyaphorophyia chalybea }\end{array}$ & F & (GC) & $\mathrm{R}$ & $x$ & - & - \\
\hline $\begin{array}{l}\text { Chestnut Wattle-eye Dyaphorophyia } \\
\text { castanea }\end{array}$ & F & (GC) & $\mathrm{R}$ & $X$ & $X$ & $X$ \\
\hline $\begin{array}{l}\text { White-spotted Wattle-eye } \\
\text { Dyaphorophyia tonsa }\end{array}$ & $\mathrm{F}$ & (GC) & $\mathrm{R}$ & $X$ & - & $X$ \\
\hline $\begin{array}{l}\text { Chestnut-capped Flycatcher } \\
\text { Erythrocercus mccalli }\end{array}$ & $\mathrm{F}$ & GC & $\mathrm{R}$ & $X$ & $X$ & $x$ \\
\hline $\begin{array}{l}\text { Dusky Crested Flycatcher Elminia } \\
\text { nigromitrata }\end{array}$ & F & (GC) & $\mathrm{R}$ & $X$ & $X$ & $X$ \\
\hline $\begin{array}{l}\text { Blue-headed Crested Flycatcher } \\
\text { Trochocercus nitens }\end{array}$ & F & (GC) & $\mathrm{R}$ & $X$ & $X$ & $X$ \\
\hline $\begin{array}{l}\text { Paradise Flycatcher Terpsiphone } \\
\text { viridis }\end{array}$ & & & $\mathrm{R}$ & $X$ & $X$ & $X$ \\
\hline $\begin{array}{l}\text { Red-bellied Paradise Flycatcher } \\
\text { Terpsiphone rufiventer }\end{array}$ & F & $(\mathrm{GC})$ & $\mathrm{R}$ & $X$ & $X$ & $X$ \\
\hline Brown Illadopsis Illadopsis fulvescens & $\mathrm{F}$ & (GC) & $\mathrm{R}$ & $x$ & $X^{*}$ & $x$ \\
\hline $\begin{array}{l}\text { Pale-breasted Illadopsis Illadopsis } \\
\text { rufipennis }\end{array}$ & F & & $\mathrm{R}$ & $X$ & $X^{*}$ & $X$ \\
\hline Blackcap Illadopsis Illadopsis cleaveri & F & GC & $\mathrm{R}$ & $x$ & $x$ & $x$ \\
\hline Dusky Tit Parus funereus & $\mathrm{F}$ & (GC) & $\mathrm{R}$ & $\mathrm{X}$ & $\mathrm{X}$ & $\mathrm{X}$ \\
\hline $\begin{array}{l}\text { Yellow-fronted Penduline Tit } \\
\text { Anthoscopus flavifrons }\end{array}$ & $\mathrm{F}$ & GC & $\mathrm{R}$ & $X$ & - & $X$ \\
\hline Tit-hylia Pholidornis rushiae & $\mathrm{F}$ & (GC) & $\mathrm{R}$ & $x$ & $X^{*}$ & $X$ \\
\hline Fraser's Sunbird Anthreptes fraseri & $\mathrm{F}$ & (GC) & $\mathrm{R}$ & $\mathrm{X}$ & $\mathrm{X}^{*}$ & $\mathrm{X}$ \\
\hline $\begin{array}{l}\text { Violet-tailed Sunbird Anthreptes } \\
\text { aurantium }\end{array}$ & $\mathrm{F}$ & GC & $\mathrm{R}$ & $\mathrm{X}$ & $X^{*}$ & $\mathrm{x}$ \\
\hline $\begin{array}{l}\text { Yellow-chinned Sunbird Anthreptes } \\
\text { rectirostris }\end{array}$ & F & (GC) & $\mathrm{R}$ & $x$ & $X^{*}$ & $X$ \\
\hline Collared Sunbird Anthreptes collaris & $\mathrm{F}$ & & $\mathrm{R}$ & $\mathrm{X}$ & $\mathrm{X}$ & $\mathrm{X}$ \\
\hline $\begin{array}{l}\text { Little Green Sunbird Nectarinia } \\
\text { seimundi }\end{array}$ & F & (GC) & $\mathrm{R}$ & $X$ & - & $X$ \\
\hline Bates's Sunbird Nectarinia batesi & $\mathrm{F}$ & (GC) & $\mathrm{R}$ & $\mathrm{x}$ & - & - \\
\hline Olive Sunbird Nectarinia olivacea & $\mathrm{F}$ & & $\mathrm{R}$ & $X$ & $x$ & $X$ \\
\hline $\begin{array}{l}\text { Reichenbach's Sunbird Nectarinia } \\
\text { reichenbachii }\end{array}$ & $\mathrm{F}$ & $(\mathrm{GC})$ & $\mathrm{R}$ & $x$ & - & $X$ \\
\hline Green-headed Sunbird Nectarinia & $\mathrm{F}$ & & $\mathrm{R}$ & - & $X$ & $X$ \\
\hline
\end{tabular}


Table 1. cont.

\begin{tabular}{|c|c|c|c|c|c|c|}
\hline Species & Forest & $\begin{array}{l}\text { Choro- } \\
\operatorname{logy}\end{array}$ & Status & Lobéké & $\begin{array}{l}\text { Dzanga- } \\
\text { Ndoki }\end{array}$ & $\begin{array}{l}\text { Nouabalé- } \\
\text { Ndoki }\end{array}$ \\
\hline $\begin{array}{l}\text { Blue-throated Brown Sunbird } \\
\text { Nectarinia cyanolaema }\end{array}$ & $\mathrm{F}$ & (GC) & $\mathrm{R}$ & $X$ & $\mathrm{X}^{*}$ & $X$ \\
\hline $\begin{array}{l}\text { Green-throated Sunbird Nectarinia } \\
\text { rubescens }\end{array}$ & $\mathrm{F}$ & (GC) & $\mathrm{R}$ & $x$ & $x$ & $x$ \\
\hline $\begin{array}{l}\text { Olive-bellied Sunbird Nectarinia } \\
\text { chloropygia }\end{array}$ & $\mathrm{F}$ & & $\mathrm{R}$ & $x$ & $x$ & $x$ \\
\hline Tiny Sunbird Nectarinia minulla & F & GC & $\mathrm{R}$ & $x$ & - & $x$ \\
\hline Coppery Sunbird Nectarinia cuprea & & & V & - & - & $x$ \\
\hline $\begin{array}{l}\text { Splendid Sunbird Nectarinia } \\
\text { coccinigaster }\end{array}$ & & & V & - & $x$ & - \\
\hline $\begin{array}{l}\text { Johanna's Sunbird Nectarinia } \\
\text { johannae }\end{array}$ & F & GC & $\mathrm{R}$ & $x$ & $X^{*}$ & $x$ \\
\hline Superb Sunbird Nectarinia superba & F & (GC) & $\mathrm{R}$ & $X$ & - & $x$ \\
\hline $\begin{array}{l}\text { Western Black-headed Oriole } \\
\text { Oriolus brachyrhynchus }\end{array}$ & F & (GC) & $\mathrm{R}$ & $x$ & $x$ & $x$ \\
\hline $\begin{array}{l}\text { Black-winged Oriole Oriolus } \\
\text { nigripennis }\end{array}$ & $\mathrm{F}$ & (GC) & $\mathrm{R}$ & $x$ & $x$ & $x$ \\
\hline $\begin{array}{l}\text { Black-shouldered Puffback } \\
\text { Dryoscopus senegalensis }\end{array}$ & $\mathrm{F}$ & GC & $\mathrm{R}$ & $x$ & - & $x$ \\
\hline Sabine's Puffback Dryoscopus sabini & F & GC & $\mathrm{R}$ & $X$ & $x$ & $x$ \\
\hline $\begin{array}{l}\text { Lühder's Bush Shrike Laniarius } \\
\text { luehderi }\end{array}$ & $\mathrm{F}$ & (GC) & $\mathrm{R}$ & - & - & $\mathrm{X}$ \\
\hline $\begin{array}{l}\text { Tropical Boubou Laniarius } \\
\text { aethiopicus }\end{array}$ & & & $\mathrm{R}$ & - & $x$ & - \\
\hline $\begin{array}{l}\text { Sooty Boubou Laniarius } \\
\text { leucorhynchus }\end{array}$ & $\mathrm{F}$ & (GC) & $\mathrm{R}$ & $x$ & $x$ & $x$ \\
\hline $\begin{array}{l}\text { Grey-green Bush Shrike Malaconotus } \\
\text { bocagei }\end{array}$ & $\mathrm{F}$ & (GC) & $\mathrm{R}$ & - & - & $x$ \\
\hline $\begin{array}{l}\text { Fiery-breasted Bush Shrike } \\
\text { Malaconotus cruentus }\end{array}$ & F & GC & $\mathrm{R}$ & $x$ & - & $x$ \\
\hline Western Nicator Nicator chloris & $\mathrm{F}$ & (GC) & $\mathrm{R}$ & $x$ & $\mathrm{X}^{*}$ & $\mathrm{X}$ \\
\hline $\begin{array}{l}\text { Yellow-throated Nicator Nicator } \\
\text { vireo }\end{array}$ & $\mathrm{F}$ & (GC) & $\mathrm{R}$ & $x$ & $X^{*}$ & $x$ \\
\hline $\begin{array}{l}\text { Northern Red-billed Helmet Shrike } \\
\text { Prionops caniceps }\end{array}$ & $\mathrm{F}$ & (GC) & $\mathrm{R}$ & $x$ & $x$ & $x$ \\
\hline Shining Drongo Dicrurus atripennis & $\mathrm{F}$ & GC & $\mathrm{R}$ & $x$ & $x$ & $x$ \\
\hline $\begin{array}{l}\text { Fork-tailed Drongo Dicrurus } \\
\text { adsimilis }\end{array}$ & F & & $\mathrm{R}$ & $x$ & - & $x$ \\
\hline $\begin{array}{l}\text { Narrow-tailed Starling Poeoptera } \\
\text { lugubris }\end{array}$ & F & (GC) & $\mathrm{R}$ & $x$ & - & - \\
\hline $\begin{array}{l}\text { Forest Chestnut-winged Starling } \\
\text { Onychognathus fulgidus }\end{array}$ & $\mathrm{F}$ & (GC) & $\mathrm{R}$ & $X$ & - & $X$ \\
\hline $\begin{array}{l}\text { Purple-headed Glossy Starling } \\
\text { Lamprotornis purpureiceps }\end{array}$ & $\mathrm{F}$ & (GC) & $\mathrm{R}$ & $x$ & $x$ & $x$ \\
\hline $\begin{array}{l}\text { Splendid Glossy Starling } \\
\text { Lamprotornis splendidus }\end{array}$ & $\mathrm{F}$ & & $\mathrm{R}$ & $x$ & $x$ & $x$ \\
\hline $\begin{array}{l}\text { Yellow-billed Oxpecker Buphagus } \\
\text { africanus }\end{array}$ & & & $\mathrm{R}$ & $x$ & $x$ & $x$ \\
\hline Grey-headed Sparrow Passer griseus & & & $\mathrm{R}$ & - & - & $x$ \\
\hline $\begin{array}{l}\text { Black-necked Weaver Ploceus } \\
\text { nigricollis }\end{array}$ & & & $\mathrm{R}$ & - & - & $x$ \\
\hline Spectacled Weaver Ploceus ocularis & & & $\mathrm{R}$ & - & $\mathrm{x}$ & - \\
\hline Orange Weaver Ploceus aurantius & $\mathrm{F}$ & (GC) & $\mathrm{R}$ & $x$ & $x$ & $x$ \\
\hline
\end{tabular}


Table 1. cont.

\begin{tabular}{|c|c|c|c|c|c|c|}
\hline Species & Forest & $\begin{array}{l}\text { Choro- } \\
\log y\end{array}$ & Status & Lobéké & $\begin{array}{l}\text { Dzanga- } \\
\text { Ndoki }\end{array}$ & $\begin{array}{l}\text { Nouabalé- } \\
\text { Ndoki }\end{array}$ \\
\hline $\begin{array}{l}\text { Vieillot's Black Weaver Ploceus } \\
\text { nigerrimus }\end{array}$ & & GC & $\mathrm{R}$ & $x$ & - & $x$ \\
\hline Village Weaver Ploceus cucullatus & & & $\mathrm{R}$ & - & - & $x$ \\
\hline $\begin{array}{l}\text { Yellow-mantled Weaver Ploceus } \\
\text { tricolor }\end{array}$ & $\mathrm{F}$ & $(\mathrm{GC})$ & $\mathrm{R}$ & $X$ & - & $X$ \\
\hline $\begin{array}{l}\text { Maxwell's Black Weaver Ploceus } \\
\text { albinucha }\end{array}$ & $\mathrm{F}$ & GC & $\mathrm{R}$ & $x$ & $X^{*}$ & $X$ \\
\hline $\begin{array}{l}\text { Preuss's Golden-backed Weaver } \\
\text { Ploceus preussi }\end{array}$ & $\mathrm{F}$ & $(\mathrm{GC})$ & $\mathrm{R}$ & $x$ & - & $x$ \\
\hline $\begin{array}{l}\text { Yellow-capped Weaver Ploceus } \\
\text { dorsomaculatus }\end{array}$ & $\mathrm{F}$ & GC & $\mathrm{R}$ & $X$ & - & $X$ \\
\hline $\begin{array}{l}\text { Blue-billed Malimbe Malimbus } \\
\text { nitens }\end{array}$ & $\mathrm{F}$ & GC & $\mathrm{R}$ & $x$ & $x$ & $x$ \\
\hline $\begin{array}{l}\text { Crested Malimbe Malimbus } \\
\text { malimbicus }\end{array}$ & $\mathrm{F}$ & $(\mathrm{GC})$ & $\mathrm{R}$ & $x$ & $x$ & $x$ \\
\hline $\begin{array}{l}\text { Cassin's Malimbe Malimbus } \\
\text { cassini }\end{array}$ & $\mathrm{F}$ & GC & $\mathrm{R}$ & $x$ & $X^{*}$ & $X$ \\
\hline $\begin{array}{l}\text { Red-headed Malimbe Malimbus } \\
\text { rubricollis }\end{array}$ & $\mathrm{F}$ & $(\mathrm{GC})$ & $\mathrm{R}$ & $x$ & $x$ & $X$ \\
\hline $\begin{array}{l}\text { Red-bellied Malimbe Malimbus } \\
\text { erythrogaster }\end{array}$ & $\mathrm{F}$ & GC & $\mathrm{R}$ & $x$ & - & $x$ \\
\hline $\begin{array}{l}\text { Red-crowned Malimbe Malimbus } \\
\text { coronatus }\end{array}$ & $\mathrm{F}$ & GC & $\mathrm{R}$ & $x$ & - & $x$ \\
\hline $\begin{array}{l}\text { Thick-billed Weaver Amblyospiza } \\
\text { albifrons }\end{array}$ & & & $\mathrm{R}$ & $x$ & $x$ & - \\
\hline $\begin{array}{l}\text { Red-headed Flower-pecker } \\
\text { Parmoptila woodhousei }\end{array}$ & $\mathrm{F}$ & (GC) & $\mathrm{R}$ & $x$ & $X^{*}$ & $X$ \\
\hline $\begin{array}{l}\text { Grey-crowned Negrofinch Nigrita } \\
\text { canicapilla }\end{array}$ & $\mathrm{F}$ & & $\mathrm{R}$ & $x$ & $x$ & $x$ \\
\hline $\begin{array}{l}\text { Pale-fronted Negrofinch Nigrita } \\
\text { luteifrons }\end{array}$ & F & (GC) & $\mathrm{R}$ & $x$ & - & $x$ \\
\hline $\begin{array}{l}\text { Chestnut-breasted Negrofinch } \\
\text { Nigrita bicolor }\end{array}$ & F & (GC) & $\mathrm{R}$ & $x$ & $X^{*}$ & $X$ \\
\hline $\begin{array}{l}\text { White-breasted Negrofinch Nigrita } \\
\text { fusconota }\end{array}$ & $\mathrm{F}$ & (GC) & $\mathrm{R}$ & $\mathrm{x}$ & $\mathrm{x}$ & $\mathrm{X}$ \\
\hline $\begin{array}{l}\text { Black-bellied Seed-cracker Pyrenestes } \\
\text { ostrinus }\end{array}$ & $\mathrm{F}$ & & $\mathrm{R}$ & $x$ & - & $X$ \\
\hline $\begin{array}{l}\text { Grant's Bluebill Spermophaga } \\
\text { poliogenys }\end{array}$ & F & GC & $\mathrm{R}$ & - & - & $x$ \\
\hline Bluebill Spermophaga haematina & $\mathrm{F}$ & (GC) & $\mathrm{R}$ & $\mathrm{x}$ & $X^{*}$ & $x$ \\
\hline $\begin{array}{l}\text { Green Twinspot Mandingoa } \\
\text { nitidula }\end{array}$ & $\mathrm{F}$ & & $\mathrm{R}$ & $x$ & - & $X$ \\
\hline Common Waxbill Estrilda astrild & & & $\mathrm{R}$ & - & $\mathrm{x}$ & - \\
\hline $\begin{array}{l}\text { Black-headed Waxbill Estrilda } \\
\text { atricapilla }\end{array}$ & & & $\mathrm{R}$ & $X$ & $x$ & $X$ \\
\hline Locust Finch Ortygospiza locustella & & & $\mathrm{R}$ & $X$ & - & - \\
\hline $\begin{array}{l}\text { Bronze Mannikin Lonchura } \\
\text { cucullata }\end{array}$ & & & $\mathrm{R}$ & - & - & $\mathrm{X}$ \\
\hline $\begin{array}{l}\text { Black-and-white Mannikin Lonchura } \\
\text { bicolor }\end{array}$ & & & $\mathrm{R}$ & $x$ & $x$ & $x$ \\
\hline Totals & 356 & & & 305 & 261 & 314 \\
\hline Forest species & 236 & & & 223 & 181 & 223 \\
\hline
\end{tabular}


sistent observation and tape playback, eventually three birds were located (two provoked into song), one in each of the three main patches of Rhynchospora marsh found at Djangé. In the absence of spontaneous vocal activity, it is not possible to give density figures for Djangé marsh, but the available habitat could hold at least 20 pairs. Rhynchospora sedges are an important constituent of most of the large marshes of Lobéké (L. Usongo, pers. comm.), and the warbler seems entirely confined to this type of vegetation (as also observed in Gabon: Christy 1994). Given the extensive habitat available (as evident in aerial photos examined) and the small size of territories, it is possible that Lobéké could hold over 100 pairs, although further surveys are necessary to confirm this. Nki and Lobéké represent a notable extension of known range to the east of some 400 $\mathrm{km}$.

\section{African Grey Parrot Psittacus erithacus}

Although not globally threatened, the status of the species gives cause for concern as its range has contracted in many regions (including several parts of Cameroon), following trapping of live birds for trade. The saline swamps of Lobéké attract many thousands of birds who come daily to seek minerals and several thousands are caught illegally each year by trappers; such numbers are likely to come from an area several times that of the Reserve, but this incessant trapping is bound to have serious consequences on the species's population dynamics. The livelihood of many people depends solely on this industry; trappers continue to operate almost at will as the local "ecoguards" (who started to patrol the area in 1999) are too few and have no authority. The bird-catchers also take Red-fronted Parrots Poicephalus gulielmi when they can, but this species is of rare occurrence in the area.

\section{Discussion}

\section{Biodiversity}

The number of 305 species recorded in Lobéké compares well with the 314 species observed in adjacent Nouabalé-Ndoki (Table 1), which benefited from similar observer coverage (Dowsett-Lemaire 1997, Dowsett-Lemaire and Dowsett 1997). Just to the west of Lobéké, we also recorded a total of 303 species in the Faunal Reserves of Boumba-Bek and Nki, in surveys adding up to six weeks (Dowsett-Lemaire and Dowsett 1998b). At Dzangha-Ndoki in RCA, the forest avifauna was studied incompletely by Green and Carroll (1991), and many species numerous in the understorey of forest in this region are absent from their list. A brief investigation in the border area between RCA and neighbouring Nouabalé-Ndoki in April 1996 (F.D.L.) added 67 mostly forest species to Green and Carroll's list in a few hours spent on the Wali stream and the Sangha near Bomassa. Knowledge of the avifauna of Dzangha-Ndoki is still too incomplete and superficial for any further comparison with that of Lobéké to be worthwhile, except to say that several species of dry savanna present in RCA are unlikely to occur in Lobéké (e.g. Senegal Coucal Centropus senegalensis). Clearly more surveys are needed in that area: in particular, key species such as the unidentified 


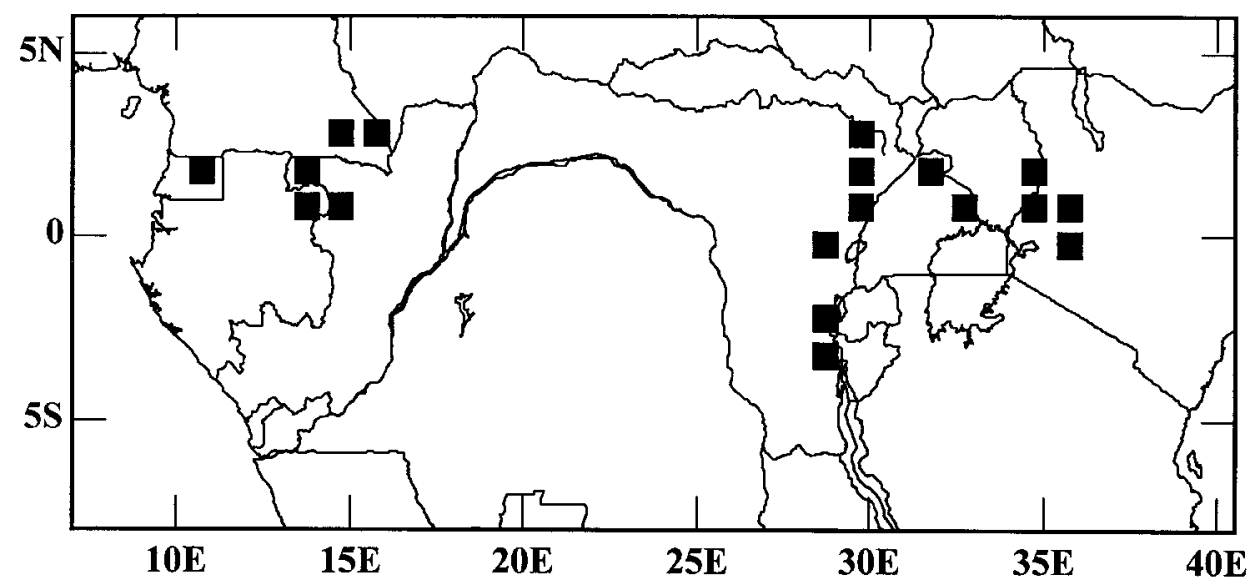

Figure 2. Known distribution of Uganda Woodland Warbler Phylloscopus budongoensis.

forest nightjar and the Dja River Warbler should be sought in suitable habitat. The occurrence of the warbler on the Congo side also remains possible, but the one swamp we saw in Nouabalé-Ndoki (Mbéli bai) did not have the right type of vegetation; other small swamps exist but are difficult of access.

Published information on Lobéké prior to our fieldwork was limited to Smith et al. (1996), who spent a week in Djangé marsh in 1992 and produced a short list of 75 species. Of these, two remain unconfirmed and are excluded from Table 1 as we consider them likely to be the result of misidentification: Red-eyed Dove Streptopelia semitorquata (for Afep Pigeon Columba unicincta, not mentioned but in fact common) and Magpie Mannikin Lonchura fringilloides (likewise for Blackand-white Mannikin L. bicolor).

The altitude of the part of Bomassa-Ndoki explored up to now is about $340 \mathrm{~m}$, and the Park in general is on average $200 \mathrm{~m}$ lower than the Lobéké Reserve. These differences in altitude explain almost certainly why some species, such as White-headed Wood Hoopoe Phoeniculus bollei, Uganda Woodland Warbler Phylloscopus budongoensis and Black-throated Apalis Apalis jacksoni, are common at Lobéké but probably absent from Ndoki. P. budongoensis has only recently been discovered in central Africa (Figure 2), occurring usually above the altitude of $400 \mathrm{~m}$ whereas the apalis is normally found above $500 \mathrm{~m}$ (see Dowsett-Lemaire and Dowsett 1996, 1998c).

\section{Conservation importance}

The total of 356 species for the three conservation areas combined (Table 1) shows clearly the potential importance of this area for the conservation of a representative fauna of western Central Africa. Of these, 185 belong to the Guineo-Congolian biome, all but 1o known from Lobéké. Lobéké may prove to be the most important site anywhere in Cameroon/Gabon for the preservation of range-restricted Bradypterus grandis (see above); the nightjar Caprimulgus sp. ?prigoginei certainly qualifies as a "Bird to watch". Capturing a forest nightjar is exceedingly difficult, 


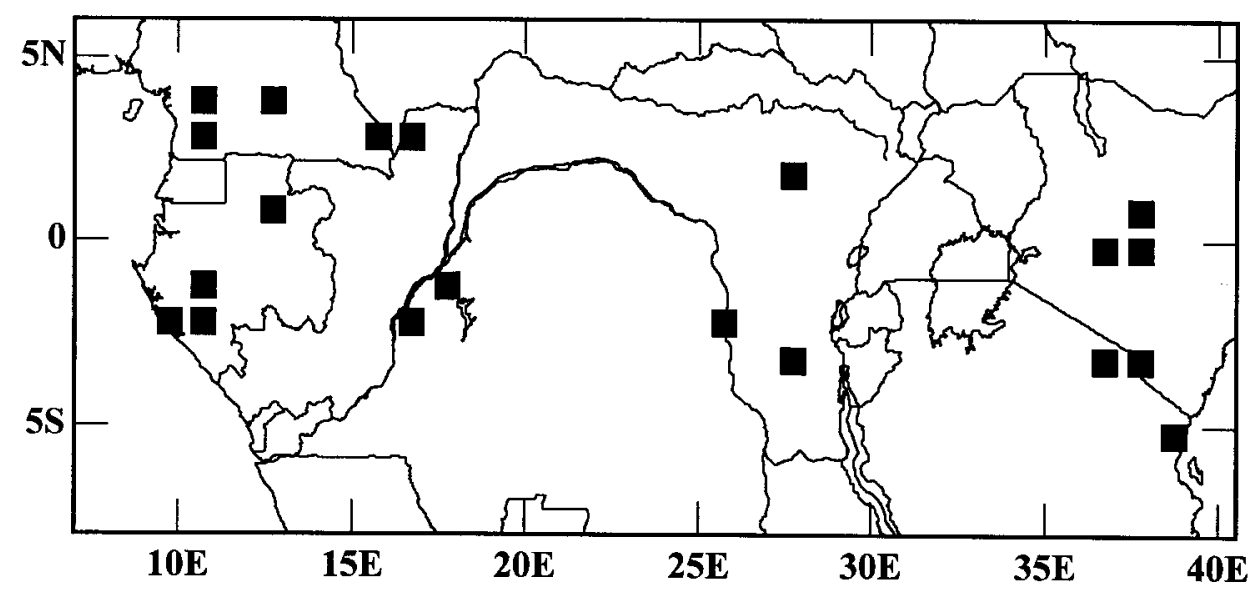

Figure 3. Eastern range of Olive Ibis Bostrychia olivacea (excluding Upper Guinea).

as unlike savanna species, they never settle on tracks and sing rather high up in trees (Dowsett-Lemaire and Dowsett 1998a). Another species of conservation concern, the Grey-necked Rockfowl Picathartes oreas, was discovered in 1998 on the eastern bank of the Boumba river (at $2^{\circ} 40^{\prime} \mathrm{N}, 15^{\circ} 13^{\prime} \mathrm{E}$ : Dowsett-Lemaire and Dowsett $1998 \mathrm{~b}$ ), thus extending its known range considerably to the east. We feel, however, that it is unlikely to be found further east in the Sangha area, as the soils of the region are very sandy and the rocky habitat needed by the Rockfowl appears to be absent.

In addition to Red Data Book species, this forested region holds an important number of birds that are generally rare throughout their range, including Olive Ibis Bostrychia olivacea, Sandy Scops Owl Otus icterorhynchus, Zenker's Honeyguide Melignomon zenkeri, Tessmann's Flycatcher Muscicapa tessmanni and Yellow-capped Weaver Ploceus dorsomaculatus. The first three have also been recorded from Boumba-Bek Faunal Reserve to the west and the flycatcher from both Boumba-Bek and Nki (Dowsett-Lemaire and Dowsett 1998b). The number of localities in Africa whence the Olive Ibis is known is not great (Figure 3), and in central Africa, it appears to be much scarcer than ill-named Spot-breasted Ibis B. rara. At Nouabalé-Ndoki we found just two pairs of the former, one near Ndoki camp and one roosting at Bomassa camp but crossing the Sangha river daily to feed on the Cameroon (Lobéké) side. Our record from Boumba-Bek is of a single bird seen in a three-week survey.

The rest of south-east Cameroon is poorly documented. Good (1953) reports a number of species collected in the region of Moloundou by himself: only a handful of these we have not found in the reserves; some are non-forest species and none is of conservation concern. Louette (1981), on the other hand, reports Bates's Weaver Ploceus batesi from "Molundu", without details; there is a specimen in the American Museum of Natural History (AMNH) collected there by Haberer in the early 1900s (Mrs M. LeCroy in litt. 1999). This is the most easterly known record. The bird is unaccountably rare throughout its small range but could conceivably occur in Lobéké. 


\section{Biogeographical considerations}

Within the forest zone, some ecological vicariants replace each other around the "Sangha interval", i.e. in the vicinity of the Sangha and Ubangui rivers. Examples of these replacements can be found in the genera Tauraco and Illadopsis. Thus two green turacos of West Africa drop out respectively just west of the Sangha river in Congo (Verreaux's Turaco Tauraco macrorhynchus, pers. obs.) and just east of the Ubangui in extreme north-west Zaire (Guinea Turaco T. persa, Snow and Louette 1981), to be replaced by Black-billed Turaco T. schuetti in the Congo basin. The Blackcap Illadopsis Illadopsis cleaveri is replaced on the other side of the Ubangui and Congo rivers by its close relative the Scaly-breasted Illadopsis I. albipectus. The Sangha itself may represent the limit of distribution of some other species more recently recorded in the region: the Black-necked Wattle-eye Dyaphorophyia chalybea, although not uncommon in Lobéké, has still not been recorded from RCA nor northern Congo. The most westerly record of the closely related Jameson's Wattle-eye D. jamesoni is from Ibembo (Schouteden 1963), so there is a gap of some $800 \mathrm{~km}$ that has neither (on present evidence). On the other hand, several Grant's Bluebills Spermophaga poliogenys were mist-netted in Nouabalé-Ndoki in 1997, a westerly extension of range. It is still unrecorded from west of the Sangha in Cameroon.

Finally, the Barred Owlet Glaucidium capense was only recently discovered in central Africa, in Ngotto in RCA (Dowsett et al. 1999), northern Congo (Dowsett-Lemaire and Dowsett 1998c) and south-east Cameroon (this survey, Dowsett and Dowsett-Lemaire in prep.). It is locally common in open-canopy forest and readily answers tapes of various races of the species. Specimens are needed to identify the race involved, but the most likely to be expected is castaneum, recorded from north-east Zaire and western Uganda (and by some considered an "incipient species"). There is no reason to suppose that the species will not in time be found in suitable habitat all along the northern edge of the Guineo-Congolian forest block in Zaire, in a continuum between the SanghaUbangui basin and north-east Zaire. This last area remains one of the least explored in central Africa.

\section{Acknowledgements}

Our surveys were undertaken at the request of WWF-Cameroon (through Dr J.S. Gartlan), and we are especially grateful to the following staff of that organization for logistical support: Dr Atanga Ekobo, Dr Leonard Usongo, Valentin Mokoguya and the tireless driver Zambo. In the field we appreciated the help and good humour of Dr P. Robinson Ngnegueu (biologist), Eloua and other assistants. We had fruitful discussion with Dr Tim Davenport about Lobéké and its birds. Mrs M. LeCroy (AMNH) kindly confirmed the existence of a specimen of Ploceus batesi.

\section{References}

Christy, P. (1994) La redécouverte de la Fauvette du Dja au Gabon. Canopée 2: 7.

Collar, N. J., Crosby, M. J. and Stattersfield, A. J. (1994) Birds to watch 2: the world list of threatened birds. Cambridge: BirdLife International (BirdLife Conserv. Series 4). 
Dowsett, R. J. and Forbes-Watson, A. D. (1993) Checklist of birds of the Afrotropical and Malagasy Regions. 1. Species limits and distribution. Liège: Tauraco Press.

Dowsett, R. J., Christy, P. and Germain, M. (1999) Additions and corrections to the avifauna of Central African Republic. Malimbus 21: 1-15.

Dowsett-Lemaire, F. (1997) The avifauna of Nouabalé-Ndoki National Park, northern Congo. Tauraco Res. Rep. 6: 111-124.

Dowsett-Lemaire, F. and Dowsett, R. J. (1996) Découverte de Phylloscopus budongoensis et autres espèces à caractère montagnard dans les forêts d'Odzala (Cuvette congolaise). Alauda 64: 364-367.

Dowsett-Lemaire, F. and Dowsett, R .J. (1997) Further investigations of the avifauna of Nouabalé-Ndoki National Park, Congo. Unpublished report of Tauraco a.s.b.l.

Dowsett-Lemaire, F. and Dowsett, R. J. (1998a) Vocal and other peculiarities of Brown Nightjar Caprimulgus binotatus. Bull. Afr. Bird Club 5: 35-38.

Dowsett-Lemaire, F. and Dowsett, R. J. (1998b) Preliminary survey of the fauna (in particular the avifauna) of the reserves of Boumba-Bek and Nki, south-eastern Cameroun. Unpublished report for WWF-Cameroon.

Dowsett-Lemaire, F. and Dowsett, R. J. (1998c) Further additions and deletions from the avifauna of Congo-Brazzaville. Malimbus 20: 15-32.

Dowsett-Lemaire, F. and Dowsett, R. J. (1999) Zoological survey (birds, amphibians) of Lobéké Faunal Reserve in April 1999, with special reference to Dja River Warbler Bradypterus grandis. Unpublished report for WWF-Cameroon.

Good, A. L. (1953) The birds of French Cameroon, II. Mem. Inst. Fr. Afr. Noire (Cameroun), Sci. Nat. 3: 1-269.

Green, A. A. and Carroll, R. W. (1991) The avifauna of Dzanga-Ndoki National Park and Dzanga-Sangha Rainforest Reserve, Central African Republic. Malimbus 13: 49-66.

Lamarque, F., Stark, M. A., Fay, J. M. and Alers, M. P. T. (1990) Chapter 19: Cameroon, Pp. 90-99 in R. East ed. Antelopes: global survey and regional action plans. 3. West and Central Africa. Gland: IUCN.

Letouzey, R. (1968) Etude phytogéographique du Cameroun. Paris: P. Lechevalier.

Louette, M. (1981) The birds of Cameroon: an annotated check-list. Verhand. Konink. Acad. Wet. 163: 1-295.

Louette, M. (1990) A new species of nightjar from Zaïre. Ibis 132: 349-353.

Ogilvie-Grant, W. R. (1917) Remarks on some recent collections of birds made by $\mathrm{Mr}$ G. L. Bates in Cameroon. Ibis (10) 5: 72-90.

Schouteden, H. (1963) La faune ornithologique des districts du Bas-Uele et du Haut-Uele. Mus. R. Afr. Centr., Doc. Zool. 4: 1-241.

Smith, T. B., Rasmussen, K. K., Whitney, K. D. and Fogiel, M. K. (1996) A preliminary survey of birds from the Lac Lobeke reserve, south-eastern Cameroon. Bird Conserv. Internat. 6: 167-174.

Snow, D. W. and Louette, M. (1981). Atlas of speciation in African non-passerine birds: addenda and corrigenda 2. Bull. Brit. Orn. Club 101: 336-339.

F. DOWSETT-LEMAIRE and R. J. DOWSETT

12 rue des Lavandes, Ganges F-3419o, France. E-mail: Dowsett@aol.com 